\title{
Polytope Novikov homology
}

\author{
Alessio Pellegriniఠ
}

\begin{abstract}
Let $M$ be a closed manifold and $\mathcal{A} \subseteq H_{\mathrm{dR}}^{1}(M)$ a polytope. For each $a \in \mathcal{A}$, we define a Novikov chain complex with a multiple finiteness condition encoded by the polytope $\mathcal{A}$. The resulting polytope Novikov homology generalizes the ordinary Novikov homology. We prove that any two cohomology classes in a prescribed polytope give rise to chain homotopy equivalent polytope Novikov complexes over a Novikov ring associated with said polytope. As applications, we present a novel approach to the (twisted) Novikov Morse Homology Theorem and prove a new polytope Novikov Principle. The latter generalizes the ordinary Novikov Principle and a recent result of Pajitnov in the abelian case.
\end{abstract}

Mathematics Subject Classification. 57R58, 53D40, 37D15, 55Nxx.

\section{Introduction}

Given a closed manifold $M$ and a cohomology class $a \in H_{\mathrm{dR}}^{1}(M)$, one can define the so-called Novikov homology HN•(a), introduced by Novikov [11,12]. Roughly speaking, $\mathrm{HN}_{\bullet}(a)$ is defined by picking a Morse representative $\alpha \in a$ and a cover on which $\alpha$ pulls back to an exact form $d \tilde{f}$, and then mimicking the definition of Morse homology using $\tilde{f}$ as the underlying Morse function. The groups HN•(a) enjoy three distinctive features

- (Novikov module) The Novikov homology HN•(a) is a finitely generated module over the so-called Novikov ring $\operatorname{Nov}(a)$.

- (Cohomology invariance) The Novikov homology HN•(a) does not depend on the choice of Morse representative $\alpha$ of the prescribed cohomology class $a$.

- (Ray invariance) Morse forms on the same positive half-ray induce identical Novikov homologies: $\mathrm{HN}_{\bullet}(r \cdot a) \cong \mathrm{HN}_{\bullet}(a)$ for all $r>0$.

The (twisted) Novikov Morse Homology Theorem says that HN.(a) is isomorphic to the twisted singular homology $H_{\bullet}(M, \underline{\operatorname{Nov}}(a)) .{ }^{1}$ Using the Novikov Morse Homology Theorem, one can thus investigate the relation between $\mathrm{HN}_{\bullet}(a)$ and $\mathrm{HN}_{\bullet}(b)$, when $a \neq b$, by studying $H_{\bullet}(M, \underline{\mathrm{Nov}}(a))$ and

\footnotetext{
${ }^{1}$ See Corollary 3.4 for a precise statement.
} 
$H_{\bullet}(M, \underline{\operatorname{Nov}}(b))$ and their respective twisted coefficient systems $\underline{\operatorname{Nov}}(a)$ and Nov $(b)$ instead. The latter is a "purely" algebraic task.

In this article, we refine the construction of Novikov homology $\mathrm{HN}_{\bullet}(a)$ and define what we call polytope Novikov homology $\mathrm{HN}_{\bullet}(a, \mathcal{A})$ by including multiple finiteness conditions imposed by a polytope $\mathcal{A}=\left\langle a_{0}, \ldots, a_{k}\right\rangle \subseteq$ $H_{\mathrm{dR}}^{1}(M)$ containing $a$. These polytope Novikov homology groups $\mathrm{HN}_{\bullet}(a, \mathcal{A})$ retain the three features of $\mathrm{HN}(a)$ mentioned above, modulo replacing $\operatorname{Nov}(a)$ by a "smaller" Novikov ring $\operatorname{Nov}(\mathcal{A})$. The Main Theorem in Sect. 2 gives a dynamical relation between $\mathrm{HN}_{\bullet}(a, \mathcal{A})$ and $\mathrm{HN}_{\bullet}(b, \mathcal{A})$, i.e., by staying in the realm of Novikov homology and not resorting to the algebraic counterpart of twisted singular homology.

Theorem. (Main Theorem ${ }^{2}$ ) For every subpolytope $\mathcal{B} \subseteq \mathcal{A}$ and two cohomology classes $a, b \in \mathcal{A}$, there exists a commutative diagram

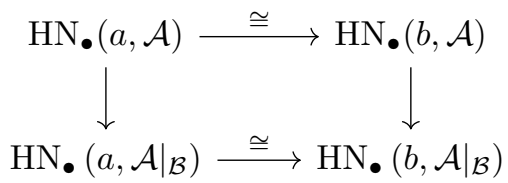

induced by continuation on the chain level.

The statement of the Main Theorem might be known to some experts in the field, but lacks a proof in the literature. Similar variants of the Main Theorem have been proved in different settings; most noteworthy are $[5,7,13$, 25]. For example, in [13], Ono considers Novikov-Floer homology on a closed symplectic manifold ${ }^{3}$ and proves the following.

Theorem. (Ono [13]) If two symplectic isotopies have fluxes that are close to each other, then their respective Novikov-Floer homologies are isomorphic.

The Novikov-Floer homologies mentioned in Ono's Theorem are defined over a common Novikov ring that takes into account several finiteness conditions simultaneously - this modification is analogous to our implementation of polytopes. Within this analogy, the upper isomorphism in the Main Theorem corresponds to the isomorphism in Ono's Theorem, but with less assumptions: the nearby assumption of the fluxes in Ono's result would translate to a smallness assumption on $\mathcal{A}$, which is not needed here. Let us mention that the formulation and setup of the Main Theorem comes closest to a recent result due to Groman and Merry [5, Theorem 5.1].

At the end of the paper, we present two applications of the Main Theorem. In the first application, we recover the aforementioned Novikov Morse Homology Theorem: ${ }^{4}$ The proof, modulo details, goes as follows: taking $\mathcal{A}=\langle 0, a\rangle$, setting $\mathcal{B}=\langle a\rangle$, invoking the lower isomorphism in the Main

\footnotetext{
${ }^{2}$ See Theorem 2.24 for the precise statement. Also note that the actual theorem contains a stronger chain-level statement.

${ }^{3}$ For the sake of simplicity, we omit the precise conditions.

${ }^{4}$ This is not a circular argument, since the Novikov Morse Homology Theorem is not used in Sect. 2.
} 
Theorem, and unwinding the definitions reveals

$$
\mathrm{HN}_{\bullet}(a) \cong \mathrm{HM}_{\bullet}(f, \underline{\mathrm{Nov}}(a)),
$$

where the right-hand side is Morse homology with local coefficients $\underline{\operatorname{Nov}}(a)$. The latter is known to be isomorphic to singular homology with twisted coefficients, for a quick proof, see [1, Theorem 4.1], and thus, we recover the Novikov Morse Homology Theorem. This line of reasoning is analogous to the proof of [5, Theorem 5.3] and seems to be a novel approach to the Novikov Morse Homology Theorem: the proof draws a direct connection between Novikov and twisted Morse homology instead of using the Novikov Principle and/or equivariant Morse homology; see $[4,8,19]$ for proofs of the Novikov Morse Homology Theorem using the latter.

The second application is concerned with a general polytope Novikov Principle: ${ }^{5}$

Theorem. (Polytope Novikov Principle) Let $\mathcal{B} \subseteq \mathcal{A}$ be a subpolytope. Then, for every $a \in \mathcal{A}$, there exists a Morse representative $\alpha \in a$, such that

$$
\mathrm{CN}_{\bullet}\left(\alpha,\left.\mathcal{A}\right|_{\mathcal{B}}\right) \simeq C_{\bullet}\left(\widetilde{M}_{\mathcal{A}}\right) \otimes_{\mathbb{Z}\left[\Gamma_{\mathcal{A}}\right]} \operatorname{Nov}\left(\left.\mathcal{A}\right|_{\mathcal{B}}\right)
$$

as Novikov modules.

The proof idea is similar to the sketch above - one relates the polytope Novikov complex to a twisted Morse complex by including the 0 -vertex in the polytope $\mathcal{A}$ and using the Main Theorem. We call this the 0 -vertex trick (cf. Lemma 3.1). To get from the twisted Morse complex to the equivariant singular chain complex, we use a Morse-Eilenberg type result (cf. Lemma 3.2 ) and a chain homotopy equivalence $\operatorname{CM}(\tilde{h}) \simeq C_{\bullet}\left(\widetilde{M}_{\mathcal{A}}\right)$ over the group ring of deck transformations $\mathbb{Z}\left[\Gamma_{\mathcal{A}}\right]$.

Immediate consequences of the polytope Novikov Principle include the ordinary Novikov Principle (cf. Corollary 3.8) and a recent "conical" Novikov Principle [17, Theorem 5.1 $]^{6}$ in the abelian case (cf. Corollary 3.10).

Remark. Symplectic homology is a version of Floer homology well suited to certain non-compact symplectic manifolds. In [18], we combine ideas of Ono's Theorem, the magnetic case [5], and of the present paper to construct a polytope Novikov symplectic homology, which is related to Ritter's twisted symplectic homology [20]. The analogue of the Main Theorem remains true. Applications include Novikov number-type bounds on the number of fixed points of symplectomorphisms with prescribed flux on the boundary, and the study of symplectic isotopies of such maps.

\section{Novikov homology and polytopes}

\subsection{Definition and properties of ordinary Novikov homology}

In this subsection, we quickly recall the (ordinary) definition of the Novikov chain complex and its homology, together with some well known properties.

\footnotetext{
${ }^{5}$ This is a slightly imprecise formulation; see Theorem 3.6 for the precise statement.

${ }^{6}$ See Theorem 3.9 for a statement below.
} 
The main purpose is to fix the notation for the remainder of the section. For a thorough treatment of Novikov homology, we recommend [4,19,22] and the recently published [1]. For more details on the construction of the Novikov ring, see for instance [6, Chapter 4].

Fix once and for all a closed smooth oriented and connected finitedimensional manifold $M$. For any Morse-Smale pair $(\alpha, g)$ one can define the Novikov chain complex

$$
\left(\mathrm{CN}_{\bullet}(\alpha, g), \partial_{\bullet}\right) \text {, }
$$

whose homology is called Novikov homology of $(\alpha, g)$

$$
\operatorname{HN}_{i}(\alpha, g):=\operatorname{ker} \partial_{i} / \operatorname{im} \partial_{i+1}, \quad i \in \mathbb{N}_{0}=\{0,1,2, \ldots\} .
$$

It is a standard fact that two Morse-Smale pairs with cohomologous Morse forms induce isomorphic Novikov homologies, and thus, we shall write HN•(a) with $a=[\alpha]$ to denote the Novikov homology of pairs $(\alpha, g)$.

Notation. Sometimes, we will also omit the $g$ in the notation of the chain complex. Moreover, Latin lowercase letters, e.g., $a, b$, will typically denote cohomology classes, while the respective lowercase Greek letters are representatives in the corresponding cohomology classes, e.g., $\alpha \in a, \beta \in b$.

Let us quickly recall the relevant definitions. Each cohomology class $a$ determines a period homomorphism $\Phi_{a}: \pi_{1}(M) \rightarrow \mathbb{R}$ defined by integrating any representative $\alpha \in a$ over loops $\gamma$ in $M^{7}{ }^{7}$ Denote by $\operatorname{ker}(a)$ the kernel of the period homomorphism $\Phi_{a}$ and let $\pi: \widetilde{M}_{a} \rightarrow M$ be the associated abelian cover, i.e., a regular covering with $\Gamma_{a}:=\operatorname{Deck}\left(\widetilde{M}_{a}\right) \cong \pi_{1}(M) / \operatorname{ker}(a)$. Then, $\alpha$ pulls back to an exact form on $\widetilde{M}_{a}$, i.e., $\pi^{*} \alpha=d \tilde{f}_{\alpha}$ for some $\tilde{f}_{\alpha} \in C^{\infty}\left(\widetilde{M}_{a}\right)$. Define

$$
V_{i}(\alpha):=\bigoplus_{\tilde{x} \in \operatorname{Crit}_{i}\left(\tilde{f}_{\alpha}\right)} \mathbb{Z}\langle\tilde{x}\rangle, \quad i \in \mathbb{N}_{0},
$$

where $\operatorname{Crit}_{i}\left(\tilde{f}_{\alpha}\right)$ denotes the critical points of $\tilde{f}_{\alpha}$ with Morse index $i$. The $i$ th Novikov chain group $\mathrm{CN}_{i}(\alpha)$ can then be defined as the downward completion of $V_{i}(\alpha)$ with respect to $\tilde{f}_{\alpha}$, which shall be denoted by

$$
\widehat{V}_{i}(\alpha)_{\tilde{f}_{\alpha}} \text { or more concisely } \widehat{V}_{i}(\alpha)_{\alpha} \text {. }
$$

Explicitly, elements $\xi \in \mathrm{CN}_{i}(\alpha)$ are infinite sums with a finiteness condition determined by $\tilde{f}_{\alpha}$ :

$\xi=\sum_{\tilde{x} \in \operatorname{Crit}_{i}\left(\tilde{f}_{\alpha}\right)} \xi_{\tilde{x}} \tilde{x} \in \mathrm{CN}_{i}(\alpha) \Longleftrightarrow \forall c \in \mathbb{R}:\left\{\tilde{x} \mid \xi_{\tilde{x}} \neq 0 \in \mathbb{Z}, \tilde{f}_{\alpha}(\tilde{x})>c\right\}$ is finite.

The boundary operator is defined by counting Novikov-Morse trajectories of $\tilde{f}_{\alpha}$

$$
\partial: \mathrm{CN}_{i}(\alpha) \rightarrow \mathrm{CN}_{i-1}(\alpha), \quad \partial \xi:=\sum_{\tilde{x}, \tilde{y}} \xi_{\tilde{x}} \cdot \# \operatorname{alg} \underline{\mathcal{M}}\left(\tilde{x}, \tilde{y} ; \tilde{f}_{\alpha}\right) \tilde{y}
$$

\footnotetext{
${ }^{7}$ Cohomologous one-forms induce the same period homomorphism by Stokes' Theorem.
} 
where

$\mathcal{M}\left(\tilde{x}, \tilde{y} ; \tilde{f}_{\alpha}\right)=\left\{\tilde{\gamma} \in C^{\infty}(\mathbb{R}, \widetilde{M}) \mid \dot{\tilde{\gamma}}+\nabla^{\tilde{g}} \tilde{f}_{\alpha}(\tilde{\gamma})=0, \tilde{\gamma}(-\infty)=\tilde{x}, \tilde{\gamma}(+\infty)=\tilde{y}\right\}$

is the usual moduli space with $\tilde{g}=\pi^{*} g$ the pullback metric. Denote $\underline{\mathcal{M}}\left(\tilde{x}, \tilde{y} ; \tilde{f}_{\alpha}\right)=\mathcal{M}\left(\tilde{x}, \tilde{y} ; \tilde{f}_{\alpha}\right) / \mathbb{R}$. Similarly, we denote by $\mathcal{M}(x, y ; \alpha)$ and

$\underline{\mathcal{M}}(x, y ; \alpha)$ the moduli spaces downstairs. The \# alg indicates the algebraic count, i.e., counting the Novikov-Morse trajectories with signs determined by a choice of orientation of the underlying unstable manifolds.

The Novikov ring $\Lambda_{\alpha}$ associated with $\alpha \in a$ is defined as the upward completion of the group ring $\mathbb{Z}\left[\Gamma_{a}\right]$ with respect to the period homomorphism $\Phi_{a}$, and therefore

$\lambda=\sum_{A \in \Gamma_{a}} \lambda_{A} A \in \Lambda_{\alpha} \Longleftrightarrow \forall c \in \mathbb{R}:\left\{A \mid \lambda_{A} \neq 0 \in \mathbb{Z}, \Phi_{a}(A)<c\right\}$ is finite.

The Novikov ring $\Lambda_{\alpha}$ does not depend on the choice of representative $\alpha \in a$, and thus, we shall write $\Lambda_{a}$. Moreover, $\Lambda_{a}$ acts on $\mathrm{CN}_{\bullet}(\alpha)$ in the obvious way. By fixing a preferred lift $\tilde{x}_{j}$ in each fiber of the finitely many zeros $x_{j} \in$ $Z(\alpha):=\{x \in M \mid \alpha(x)=0\}$, one can view $\mathrm{CN}_{\bullet}(\alpha)$ as a finitely generated $\Lambda_{a}$-module

$$
\mathrm{CN}_{i}(\alpha) \cong \bigoplus_{x_{j} \in Z(\alpha)} \Lambda_{a}\left\langle\tilde{x}_{j}\right\rangle \text { as Novikov ring modules. }
$$

Another standard fact asserts that the boundary operator $\partial$ is $\Lambda_{a}$-linear, and consequently, the Novikov homology $\mathrm{HN}_{\bullet}(a)$ carries a $\Lambda_{a}$-module structure. The latter is implicitly using the fact that isomorphism of Novikov homologies for cohomologous Morse forms, which is suppressed in the notation $\mathrm{HN}_{\bullet}(a)$, is also $\Lambda_{a}$-linear.

Remark 2.1. If $M$ is not orientable, one can still define a Novikov homology by replacing $\mathbb{Z}$ with $\mathbb{Z}_{2}$ in all the definitions above.

\subsection{Novikov homology with polytopes}

We are now ready to refine the Novikov chain complex using polytopes - this notion is key for the proofs of all incoming theorems.

Definition 2.2. Given $a_{0}, \ldots, a_{k} \in H_{\mathrm{dR}}^{1}(M)$, denote by

$$
\mathcal{A}=\left\langle a_{0}, \ldots, a_{k}\right\rangle \subset H_{\mathrm{dR}}^{1}(M)
$$

the polytope spanned by the vertices $\left\{a_{l}\right\}_{l=0, \ldots, k}$, i.e., the set of all convex combinations

$$
a=\sum_{l=0}^{k} c_{l} \cdot a_{l} \text { with } c_{l} \in[0,1] \text { and } \sum_{l=0}^{k} c_{l}=1 .
$$

To any polytope $\mathcal{A}$, we associate a regular cover

$$
\pi: \widetilde{M}_{\mathcal{A}} \rightarrow M \text { determined by } \operatorname{Deck}\left(\widetilde{M}_{\mathcal{A}}\right) \cong \pi_{1}(M) / \bigcap_{l=0}^{k} \operatorname{ker}\left(a_{l}\right) \text {, }
$$


and we shall abbreviate

$$
\Gamma_{\mathcal{A}}:=\operatorname{Deck}\left(\widetilde{M}_{\mathcal{A}}\right)
$$

Example 2.3. For the polytope $\mathcal{A}=\langle a\rangle$, the covering $\widetilde{M}_{\mathcal{A}}$ agrees with the abelian cover $\widetilde{M}_{a}$ associated with $a \in H_{\mathrm{dR}}^{1}(M)$. The same is true for any polytope $\mathcal{A}$, whose other vertices $a_{l}$ satisfy $\operatorname{ker}(a) \subseteq \operatorname{ker}\left(a_{l}\right)$.

The defining condition of $\widetilde{M}_{\mathcal{A}}$ ensures that each vertex $a_{l}$ pulls back to the trivial cohomology class, and so does every $a \in \mathcal{A}$; see Lemma 2.7. We write $\tilde{f}_{\alpha} \in C^{\infty}\left(\widetilde{M}_{\mathcal{A}}\right)$ to denote some primitive of $\pi^{*} \alpha$ for $\alpha$ a representative of $a \in \mathcal{A}$. Now, we fix a smooth section

$$
\theta: \mathcal{A} \longrightarrow \Omega^{1}(M), \quad a \mapsto \theta_{a}
$$

of the projection of closed one-forms to their cohomology class. In other words, $\theta_{a}$ is a representative of $a$. This enables us to talk about a "preferred" representative of each cohomology class in the polytope.

For every polytope $a \in \mathcal{A}$, we define

$$
V_{i}\left(\theta_{a}, \mathcal{A}\right):=\bigoplus_{\tilde{x} \in \operatorname{Crit}_{i}\left(\tilde{f}_{\theta_{a}}\right)} \mathbb{Z}\langle\tilde{x}\rangle .
$$

The subtle but crucial difference to $V_{i}\left(\theta_{a}\right)$ is that $\widetilde{M}_{\mathcal{A}}$ does not necessarily coincide with the abelian cover $\widetilde{M}_{a}$.

Definition 2.4. Let $\mathcal{A}$ be a polytope with section $\theta: \mathcal{A} \rightarrow \Omega^{1}(M)$. Then, the (polytope) Novikov chain complex groups

$$
\mathrm{CN}_{i}\left(\theta_{a}, \mathcal{A}\right), \quad i \in \mathbb{N}_{0}
$$

are defined as the intersections of the downward completions of $V_{i}\left(\theta_{a}, \mathcal{A}\right)$ with respect to any $\tilde{f}_{\beta}: \widetilde{M}_{\mathcal{A}} \rightarrow \mathbb{R}$ for $b \in \mathcal{A}$. In other words, with the notation of (1)

$$
\mathrm{CN}_{i}\left(\theta_{a}, \mathcal{A}\right):=\bigcap_{b \in \mathcal{A}} \widehat{V}_{i}\left(\theta_{a}, \mathcal{A}\right)_{\beta}
$$

Remark 2.5. Let $\beta \in b$ be any representative. The choice of primitive $\tilde{f}_{\beta}$ of $\pi^{*} \beta$ is unique up to adding constants and hence does not affect the finiteness condition. Additionally, two primitives $\tilde{f}_{\beta}$ and $\tilde{f}_{\beta^{\prime}}$ induce the same finiteness condition for $\beta, \beta^{\prime} \in b$. Indeed, $\tilde{f}_{\beta^{\prime}}-\tilde{f}_{\beta}=h \circ \pi$ for some smooth $h: M \rightarrow \mathbb{R}$ with $d h=\beta^{\prime}-\beta$. Since $M$ is compact, we get

$\tilde{f}_{\beta}(\tilde{x})>c \Longrightarrow \tilde{f}_{\beta^{\prime}}(\tilde{x})>\min _{z} h(z)+c$ and $\tilde{f}_{\beta^{\prime}}(\tilde{x})>d \Longrightarrow \tilde{f}_{\beta}(\tilde{x})>d-\max _{z} h(z) l$

two finiteness conditions are equivalent. This justifies Definition 2.4.

Unpacking Definition 2.4, we see

$$
\begin{aligned}
\xi= & \sum_{\tilde{x} \in \operatorname{Crit}_{i}\left(\tilde{f}_{\theta_{a}}\right)} \xi_{\tilde{x}} \tilde{x} \in \mathrm{CN}_{i}\left(\theta_{a}, \mathcal{A}\right) \Longleftrightarrow \forall b \in \mathcal{A}, \forall c \in \mathbb{R}: \\
& \#\left\{\tilde{x} \mid \xi_{\tilde{x}} \neq 0, \tilde{f}_{\beta}(\tilde{x})>c\right\}<+\infty
\end{aligned}
$$


where it does not matter which primitives $\tilde{f}_{\beta}$ we use, cf. Remark 2.5. The right-hand side describes a finiteness condition that has to hold for all $b \in \mathcal{A}$, and hence, we will refer to it as the multi finiteness condition.

Notation. In view of Remark 2.5, we shall write

$$
\mathrm{CN}_{i}\left(\theta_{a}, \mathcal{A}\right)=\bigcap_{b \in \mathcal{A}} \widehat{V}_{i}\left(\theta_{a}, \mathcal{A}\right)_{b}
$$

from now on.

In a similar fashion, we can define yet another completion of $V_{i}\left(\theta_{a}, \mathcal{A}\right)$ by taking the completion with respect to less one-forms.

Definition 2.6. Let $\mathcal{B} \subseteq \mathcal{A}$ be a subpolytope, i.e., the convex hull of a subset of the vertices of $\mathcal{A}$. Then, we define

$$
\mathrm{CN}_{i}\left(\theta_{a},\left.\mathcal{A}\right|_{\mathcal{B}}\right):=\bigcap_{b \in \mathcal{B}} \widehat{V}_{i}\left(\theta_{a}, \mathcal{A}\right)_{b}
$$

the restricted (polytope) Novikov chain complex groups of $\mathcal{B} \subseteq \mathcal{A}$.

By definition, we get the inclusion

$$
\mathrm{CN}_{\bullet}\left(\theta_{a}, \mathcal{A}\right) \subseteq \mathrm{CN}_{\bullet}\left(\theta_{a},\left.\mathcal{A}\right|_{\mathcal{B}}\right), \text { for all subpolytopes } \mathcal{B} \subseteq \mathcal{A} .
$$

The next lemma asserts that $\mathrm{CN}_{\bullet}\left(\theta_{a}, \mathcal{A}\right)$ is uniquely determined by the vertices of $\mathcal{A}$. In other words, one only needs to check the multi-finiteness condition for the finitely many vertices $a_{l}$. This is a straightforward adaptation of [25, Lemma 7.3].

Lemma 2.7. Let $\theta: \mathcal{A} \rightarrow \Omega^{1}(M)$ be as above. Then

$$
\mathrm{CN}_{i}\left(\theta_{a}, \mathcal{A}\right)=\bigcap_{b \in \mathcal{A}} \widehat{V}_{i}\left(\theta_{a}, \mathcal{A}\right)_{b}=\bigcap_{l=0}^{k} \widehat{V}_{i}\left(\theta_{a}, \mathcal{A}\right)_{a_{l}}=\bigcap_{l=0}^{k} \mathrm{CN}_{i}\left(\theta_{a},\left.\mathcal{A}\right|_{a_{l}}\right), \quad \forall a \in \mathcal{A} .
$$

More generally, for every subpolytope $\mathcal{B} \subseteq \mathcal{A}$ spanned by $b_{j}=a_{l_{j}}$

$$
\mathrm{CN}_{i}\left(\theta_{a},\left.\mathcal{A}\right|_{\mathcal{B}}\right)=\bigcap_{j} \mathrm{CN}_{i}\left(\theta_{a},\left.\mathcal{A}\right|_{b_{j}}\right), \quad \forall a \in \mathcal{A} .
$$

One can play a similar game with the Novikov rings:

Definition 2.8. Define the (polytope) Novikov ring

$$
\Lambda_{\mathcal{A}}=\bigcap_{b \in \mathcal{A}} \widehat{\mathbb{Z}}\left[\Gamma_{\mathcal{A}}\right]^{b}
$$

where $\widehat{\mathbb{Z}}\left[\Gamma_{\mathcal{A}}\right]^{b}$ denotes the upward completion of the group ring $\mathbb{Z}\left[\Gamma_{\mathcal{A}}\right]$ with respect to the period homomorphism $\Phi_{b}: \Gamma_{\mathcal{A}} \rightarrow \mathbb{R}$. Analogously, for every subpolytope $\mathcal{B} \subseteq \mathcal{A}$, we define the restricted polytope Novikov ring

$$
\Lambda_{\left.\mathcal{A}\right|_{\mathcal{B}}}=\bigcap_{b \in \mathcal{B}} \widehat{\mathbb{Z}}\left[\Gamma_{\mathcal{A}}\right]^{b}
$$


As before, we get

$$
\Lambda_{\mathcal{A}} \subseteq \Lambda_{\left.\mathcal{A}\right|_{\mathcal{B}}} \text {, for all subpolytopes } \mathcal{B} \subseteq \mathcal{A} \text {. }
$$

The obvious analogue to Lemma 2.7 holds for Novikov rings as well. These rings enable us to view the polytope Novikov chain complexes as finite Novikov modules just as in the ordinary setting (2).

Next, we try to equip the groups $\mathrm{CN}_{\bullet}\left(\theta_{a}, \mathcal{A}\right)$ with a boundary operators that turns them into a genuine chain complex. The obvious candidate would be

$$
\partial_{\theta_{a}}: \mathrm{CN}_{\bullet}\left(\theta_{a}, \mathcal{A}\right) \rightarrow \mathrm{CN}_{\bullet-1}\left(\theta_{a}, \mathcal{A}\right), \quad \partial_{\theta_{a}} \xi:=\sum_{\tilde{x}, \tilde{y}} \xi_{\tilde{x}} \cdot \# \operatorname{alg} \underline{\mathcal{M}}\left(\tilde{x}, \tilde{y} ; \tilde{f}_{\theta_{a}}\right) \tilde{y}
$$

Note that the moduli space above actually also depends on a choice of metric $g$, and so does the boundary operator $\partial_{\theta_{a}}$. When we want to keep track of the metric, we will write $\mathrm{CN}_{\bullet}\left(\theta_{a}, g, \mathcal{A}\right)$. For restrictions $\left.\mathcal{A}\right|_{\mathcal{B}}$, we define the boundary operator analogously.

Formally, the definition of $\partial_{\theta_{a}}$ looks identical to the definition of $\partial$ on $\mathrm{CN}_{\bullet}(\alpha)$, and morally it is. However, there are two major differences. First, the cover $\widetilde{M}_{\mathcal{A}}$ might differ from the abelian cover $\widetilde{M}_{a}$ of $a$. Second, it is not clear whether $\partial=\partial_{\theta_{a}}$ preserves the multi finiteness condition, i.e., whether $\partial \xi$ lies in $\mathrm{CN}_{\bullet}\left(\theta_{a}, \mathcal{A}\right)$. Luckily, we will achieve this by replacing the original section $\theta$ with a perturbed section $\vartheta: \mathcal{A} \rightarrow \Omega^{1}(M)$ (cf. Theorem 2.14). Whenever the chain complex is defined, we make the following definition.

Definition 2.9. Let $\vartheta: \mathcal{A} \rightarrow \Omega^{1}(M)$ be a section, such that $\left(\mathrm{CN}_{\bullet}\left(\vartheta_{a}, g_{\vartheta_{a}}, \mathcal{A}\right), \partial\right)$ defines a chain complex. Then, we call the induced homology (polytope) Novikov homology and denote it by

$$
\mathrm{HN}_{\bullet}\left(\vartheta_{a}, g_{\vartheta_{a}}, \mathcal{A}\right) \text { or more abusively } \mathrm{HN}_{\bullet}\left(\vartheta_{a}, \mathcal{A}\right) \text {. }
$$

Analogously, we define

$$
\mathrm{HN}_{\bullet}\left(\vartheta_{a}, g_{\vartheta_{a}},\left.\mathcal{A}\right|_{\mathcal{B}}\right)=\mathrm{HN}_{\bullet}\left(\vartheta_{a},\left.\mathcal{A}\right|_{\mathcal{B}}\right) \text {. }
$$

Remark 2.10. Analogously to ordinary Novikov homology, one can show that the Novikov homologies $\mathrm{HN}_{\bullet}\left(\vartheta_{a}, \mathcal{A}\right)$ and $\mathrm{HN}_{\bullet}\left(\vartheta_{a},\left.\mathcal{A}\right|_{\mathcal{B}}\right)$ are both finitely generated modules over the Novikov rings $\Lambda_{\mathcal{A}}$ and $\Lambda_{\left.\mathcal{A}\right|_{\mathcal{B}}}$, respectively, thus generalizing the Novikov-module property. This follows from the fact that the boundary operator (4) is $\Lambda_{\mathcal{A}}$-linear (and similarly for the restricted case).

\subsection{Technical results for Sect. 2.4}

In this subsection, we state and prove all the technical auxiliary results needed for the proof of Theorem 2.14, which roughly speaking asserts the welldefinedness of the polytope chain complexes and their respective homologies after modifying the section $\theta: \mathcal{A} \rightarrow \Omega^{1}(M)$ to a new section $\vartheta: \mathcal{A} \rightarrow \Omega^{1}(M)$. Notation. For any (closed) one-form $\rho$, we will denote by $\nabla^{g} \rho$ the dual vector field to $\rho$ with respect to the metric $g$. Note that with this notation, we have $\nabla^{g} H=\nabla^{g} d H$ for any smooth function $H: M \rightarrow \mathbb{R}$. 
Proposition 2.11. Let $(\rho, g)$ be a Morse-Smale pair. Then, for every $\delta>0$, there exists a constant $C_{\rho}=C_{\rho}(\delta, g)>0$, such that

$$
\left\|\nabla^{g} \rho(z)\right\|<C_{\rho} \Longrightarrow \exists x \in Z(\rho) \text { with } d(x, z)<\delta,
$$

where both $\|\cdot\|$ and $d(\cdot, \cdot)$ are induced by $g$.

Proof. Suppose the assertion does not hold. Then, there exists a $\delta>0$, a positive sequence $C_{k} \rightarrow 0$, and $\left(z_{k}\right) \subset M$, such that

$$
\left\|\nabla^{g} \rho\left(z_{k}\right)\right\|<C_{k} \quad \text { and } \quad z_{k} \in M \backslash \bigcup_{x \in Z(\rho)} B_{\delta}(x) .
$$

By compactness of $M$, we can pass to a subsequence $\left(z_{k}\right)$ converging to some $z \in M$. The above however implies $\left\|\nabla^{g} \rho(z)\right\|=0$, which is equivalent to $z \in Z(\rho)$. At the same time, $z$ lies in $M \backslash \bigcup_{x \in Z(\rho)} B_{\delta}(x)$, which is a contradiction. This concludes the proof.

Notation. Such a constant $C_{\rho}>0$ is often referred to as a Palais-Smale constant (short: PS-constant). The main case of interest is the exact one, i.e., $\rho=d H$, for which we will abbreviate $C_{d H}=C_{H}$. Sometimes, we will also abbreviate $C_{\rho}=C$.

The next lemma builds the main technical tool of Sect. 2.4. The idea is to perturb one-forms $\alpha$ close to a given reference Morse-Smale pair $(\rho, g)$, so that the pertubations, say $\alpha^{\prime}$, maintain their cohomology classes of $\alpha$, become Morse, have the same zeros as $\rho$, and are still relatively close to $\rho$. This is reminiscent of Zhang's arguments [25, Section 3].

Lemma 2.12. Let $(\rho, g)$ be a Morse-Smale pair, $\delta>0$ so small that the balls $B_{2 \delta}(x)$, with $x \in Z(\rho)$, are geodesically convex ${ }^{8}$ and lie in pairwise disjoint charts of $M$, and $C=C_{\rho}(\delta, g)>0$ as in Proposition 2.11.

Let $\alpha \in \Omega^{1}(M)$ with

$$
\|\alpha-\rho\|<\frac{C}{8} \quad \text { and } \quad a=[\alpha]
$$

where $\|\cdot\|$ is the norm induced by $g$. Then, there exists a Morse-Smale pair $\left(\alpha^{\prime}, g^{\prime}\right)$, with $\alpha^{\prime} \in a$, satisfying

- $\left\|\alpha^{\prime}-\rho\right\| \leq 5 \cdot\|\alpha-\rho\|$,

- $\left.\alpha^{\prime}\right|_{B_{\delta}(x)}=\left.\rho\right|_{B_{\delta}(x)}$ for all $x \in Z(\rho)$ and

- $Z\left(\alpha^{\prime}\right)=Z(\rho)$.

Moreover, $\left\|\nabla^{g^{\prime}} \alpha^{\prime}(z)\right\|^{\prime}<\frac{C}{8}$ implies $z \in B_{\delta}(x)^{9}$ for some zero $x \in Z\left(\alpha^{\prime}\right)$, where $\|\cdot\|^{\prime}$ is the norm induced by $g^{\prime}$.

Proof. Since $\rho$ is a Morse form, there are only finitely many zeros $x \in Z(\rho)$. Around each such $x$, we will perturb $\alpha$ without changing its cohomology

\footnotetext{
${ }^{8}$ This is a well-known result in Riemannian geometry; see [24].

${ }^{9}$ This is still the ball of radius $\delta$ with respect to the distance metric induced by $g$.
} 
class: enumerate the finitely many zeros of $\rho$ by $\left\{x_{i}\right\}_{i=1, \ldots, k}$ and pick positive bump functions $h_{i}: M \rightarrow \mathbb{R}_{\geq 0}$ with

$$
\begin{cases}h_{i} \equiv 0, & \text { on } M \backslash B_{2 \delta}\left(x_{i}\right) \\ h_{i} \equiv 1, & \text { on } B_{\delta}\left(x_{i}\right) \\ \left\|\nabla^{g} h_{i}\right\| \leq \frac{2}{\delta} . & \end{cases}
$$

Since every $B_{2 \delta}\left(x_{i}\right)$ is simply connected, there exist unique smooth functions $f_{i}: B_{2 \delta}\left(x_{i}\right) \rightarrow \mathbb{R}$ satisfying

$$
f_{i}\left(x_{i}\right)=0 \quad \text { and } \quad d f_{i}=\left.(\alpha-\rho)\right|_{B_{2 \delta}\left(x_{i}\right)} .
$$

We set

$$
\alpha^{\prime}=\alpha-\sum_{i=1}^{k} d\left(h_{i} \cdot f_{i}\right) .
$$

By construction, we have $\alpha^{\prime} \in a, \alpha^{\prime}=\rho$ on $B_{\delta}(x)$ for $x \in Z(\rho)$, and that $\alpha^{\prime}$ agrees with $\alpha$ outside of $\bigcup_{i=0}^{k} B_{2 \delta}\left(x_{i}\right)$. Consequently, $\alpha^{\prime}-\rho$ and $\alpha-\rho$ agree outside of $\bigcup_{i=0}^{k} B_{2 \delta}\left(x_{i}\right)$. This means that for the inequality in the first bullet point, it suffices to argue why the bound holds inside each ball $B_{2 \delta}\left(x_{i}\right)$. Inserting the definitions grants

$$
\begin{aligned}
\left\|\alpha^{\prime}-\rho\right\|_{B_{2 \delta}\left(x_{i}\right)} & =\left\|\alpha-\rho-h_{i} \cdot d f_{i}-f_{i} \cdot d h_{i}\right\|_{B_{2 \delta}\left(x_{i}\right)} \\
& \leq\left(1-h_{i}\right)\|\alpha-\rho\|_{B_{2 \delta}\left(x_{i}\right)}+\left\|f_{i}\right\|_{B_{2 \delta}\left(x_{i}\right)} \cdot\left\|\nabla^{g} h_{i}\right\|_{B_{2 \delta}\left(x_{i}\right)} \\
& \leq\|\alpha-\rho\|_{B_{2 \delta}\left(x_{i}\right)}+\frac{2}{\delta} \cdot\left\|f_{i}\right\|_{B_{2 \delta}\left(x_{i}\right)} .
\end{aligned}
$$

Recall that $f_{i}$ was chosen, such that $f_{i}\left(x_{i}\right)=0$. Due to the geodesic convexity of the balls $B_{2 \delta}\left(x_{i}\right)$, we can apply the mean value inequality

$$
\begin{gathered}
\left|f_{i}(y)\right|=\left|f_{i}\left(x_{i}\right)-f_{i}(y)\right| \leq\left\|\nabla^{g} f_{i}\right\|_{B_{2 \delta}\left(x_{i}\right)} \cdot d\left(x_{i}, y\right) \leq\|\alpha-\rho\|_{B_{2 \delta}\left(x_{i}\right)} \cdot 2 \delta, \\
\forall y \in B_{2 \delta}\left(x_{i}\right) .
\end{gathered}
$$

All in all, this implies

$$
\left\|\alpha^{\prime}-\rho\right\|_{B_{2 \delta}\left(x_{i}\right)} \leq\|\alpha-\rho\|_{B_{2 \delta}\left(x_{i}\right)}+\frac{4 \delta}{\delta}\|\alpha-\rho\|_{B_{2 \delta}\left(x_{i}\right)}=5 \cdot\|\alpha-\rho\|_{B_{2 \delta}\left(x_{i}\right)} .
$$

This proves the first inequality in the first bullet point. From this, we will deduce that $Z(\rho)=Z\left(\alpha^{\prime}\right)$ : the inclusion $Z(\rho) \subseteq Z\left(\alpha^{\prime}\right)$ is clear as $\rho$ agrees with $\alpha^{\prime}$ around $Z(\rho)$. The reverse inclusion is obtained by observing that for $y \in Z\left(\alpha^{\prime}\right)$, we have

$$
\left\|\nabla^{g} \rho(y)\right\|=\|\nabla^{g} \rho(y)-\underbrace{\nabla^{g} \alpha^{\prime}(y)}_{=0}\| \leq\left\|\rho-\alpha^{\prime}\right\| \leq 5 \cdot\|\alpha-\rho\|<C,
$$

by assumption on $\rho$ and the inequality above. Proposition 2.11 then implies that $z$ has to be a zero of $\rho$, as well. This proves $Z(\rho)=Z\left(\alpha^{\prime}\right)$, in particular that $\alpha^{\prime}$ is a Morse form.

To get a Riemannian metric $g^{\prime}$ that turns $\left(\alpha^{\prime}, g^{\prime}\right)$ into a Morse-Smale pair, it suffices to perturb $g$ on an open set that intersects all the NovikovMorse trajectories of $\left(\alpha^{\prime}, g\right)$; see [19, Page 38-40] for more details. Since 
$Z(\rho)=Z\left(\alpha^{\prime}\right)$, we can take a perturbation $g^{\prime}$ that agrees with $g$ on $M \backslash$ $\bigcup_{i=1}^{k} B_{\delta}\left(x_{i}\right)$ and is close to $g$ in the $C^{\infty}$-topology.

The last assertion of the statement follows from the observation that, for $\alpha^{\prime}$ fixed, the map $g^{\prime} \mapsto\left\|\nabla^{g^{\prime}} \alpha^{\prime}\right\|^{\prime}$ is continuous, and thus, for $g^{\prime}$ close to $g$, we get

$$
\left\|\nabla^{g} \alpha^{\prime}(z)\right\| \leq \underbrace{\left\|\nabla^{g} \alpha^{\prime}(z)\right\|-\left\|\nabla^{g^{\prime}} \alpha^{\prime}(z)\right\|^{\prime} \mid}_{<\varepsilon}+\left\|\nabla^{g^{\prime}} \alpha^{\prime}(z)\right\|^{\prime} .
$$

Assuming $\left\|\nabla^{g^{\prime}} \alpha^{\prime}(z)\right\|^{\prime}<\frac{C}{8}$, we thus end up with

$$
\begin{aligned}
\left\|\nabla^{g} \rho(z)\right\| & \leq\left\|\nabla^{g} \rho(z)-\nabla^{g} \alpha^{\prime}(z)\right\|+\left\|\nabla^{g} \alpha^{\prime}(z)\right\| & & \\
& \leq\left\|\rho-\alpha^{\prime}\right\|+\varepsilon+\frac{C}{8} & & \text { using the above inequalit }, \\
& \leq 5 \cdot\|\rho-\alpha\|+\varepsilon+\frac{C}{8} & & \text { by the first bullet point, } \\
& <\frac{5 \cdot C}{8}+\varepsilon+\frac{C}{8} & & \text { by assumption. }
\end{aligned}
$$

Taking $\varepsilon \leq \frac{C}{4}$ and invoking Proposition 2.11 then conclude the proof.

Lemma 2.12 can be applied to a whole section $\theta: \mathcal{A} \rightarrow \Omega^{1}(M)$ nearby a reference Morse-Smale pair $(\rho, g)$ and give rise to a perturbed section $\vartheta: \mathcal{A} \rightarrow$ $\Omega^{1}(M)$ that is still relatively close to $\rho$, so that each $\vartheta_{a}$ agrees with $\rho$ near the zeros $x \in Z(\rho)$.

Proposition 2.13. Let $(\rho, g)$ and $C=C_{\rho}>0$ as in Lemma 2.12, denote $N=$ $\bigcup_{i} B_{2 \delta}\left(x_{i}\right)$ with $x_{i} \in Z(\rho)$, and let $\theta: \mathcal{A} \rightarrow \Omega^{1}(M)$ be a section satisfying

$$
\left\|\theta_{a}-\rho\right\|<\frac{C}{8} \text {. }
$$

Then, there exists a section

$$
\vartheta=\vartheta(\theta, \rho, g): \mathcal{A} \longrightarrow \Omega^{1}(M)
$$

and a positive constant $D=D(N, g)>0$ with the following significance: ${ }^{10}$

- $Z\left(\vartheta_{a}\right)=Z(\rho)$ for all $a \in \mathcal{A}$,

- $\left.\vartheta_{a}\right|_{B_{\delta}\left(x_{i}\right)}=\left.\rho\right|_{B_{\delta}\left(x_{i}\right)}$ for all $x_{i} \in Z(\rho), a \in \mathcal{A}$.

Moreover, for every $\vartheta_{a}$, there exists a Riemannian metric $g_{\vartheta_{a}}$ close to $g$ with $\left.g_{\vartheta_{a}}\right|_{M \backslash N}=\left.g\right|_{M \backslash N}$, such that

- $\left(\vartheta_{a}, g_{\vartheta_{a}}\right)$ is Morse-Smale,

- $\left\|\vartheta_{b}-\rho\right\|_{\vartheta_{a}} \leq D \cdot\left\|\vartheta_{b}-\rho\right\| \leq 5 \cdot D \cdot\left\|\theta_{b}-\rho\right\|$ for all $a, b \in \mathcal{A}$,

where $\|\cdot\|_{\vartheta_{a}}$ is the operator norm induced by $g_{\vartheta_{a}}$.

Proof. Since the whole section $\theta: \mathcal{A} \rightarrow \Omega^{1}(M)$ is $\frac{C}{8}$-close to $(\rho, g)$, we can take $(\rho, g)$ as a reference pair and apply Lemma 2.12 to every $\theta_{a}$ and denote $\vartheta_{a}$ the corresponding perturbation. Recall from the proof of Lemma 2.12 that $\vartheta_{a}$ is obtained by an exact perturbation of $\theta_{a}$ around the zeros of $\rho$-a closer inspection reveals that this exact perturbation varies smoothly along $\theta_{a}$, in

${ }^{10}$ The choice of $D>0$ is independent of the assumption (7). 
particular that $\vartheta$ defines a smooth section. The first two bullet points follow immediately from Lemma 2.12.

We choose $g_{\vartheta_{a}}=g_{\left(\theta_{a}\right)^{\prime}}$ just as $g^{\prime}$ in Lemma 2.12, i.e., by means of a small perturbation of $g$ inside $N$. The argument in [19] shows that sufficiently small perturbations give rise to Riemannian metrics that are uniformly equivalent to the original $g$; in other words, we may choose $g_{\vartheta_{a}}$, such that $\left(\vartheta_{a}, g_{\vartheta_{a}}\right)$ is Morse-Smale and

$$
\frac{1}{D^{2}} g_{\vartheta_{a}}(v, v) \leq g(v, v) \leq D^{2} g_{\vartheta_{a}}(v, v), \quad \forall v \in T M, \forall a \in \mathcal{A},
$$

with $D>0$ a constant that only depends on $N$ and $g$. Using this inequality and invoking, the first bullet point of Lemma 2.12 conclude the proof.

\subsection{Section perturbations}

We can finally state and prove Theorem 2.14 by applying the previous results in the special case of exact reference pairs:

Theorem 2.14. Let $\theta: \mathcal{A} \rightarrow \Omega^{1}(M)$ be a section and $(H, g)$ a reference MorseSmale pair on $M$. Then, there exists a perturbed section

$$
\vartheta=\vartheta(\theta, H, g): \mathcal{A} \longrightarrow \Omega^{1}(M)
$$

and a choice of Riemannian metrics $g_{\vartheta_{a}}$ with the following significance:

- (Morse-Smale property) Each pair $\left(\vartheta_{a}, g_{\vartheta_{a}}\right)$ is Morse-Smale, for all $a \in \mathcal{A}$;

- (Chain complex) The chain complex $\left(\mathrm{CN} \bullet\left(\vartheta_{a}, g_{\vartheta_{a}}, \mathcal{A}\right), \partial_{\vartheta_{a}}\right)$ is well defined for every pair $\left(\vartheta_{a}, g_{\vartheta_{a}}\right)$ as above;

- (Ray invariance) The chain complexes are equal upon scaling, i.e., $\mathrm{CN}_{\bullet}\left(\vartheta_{a}, g_{\vartheta_{a}}, \mathcal{A}\right)=\mathrm{CN}_{\bullet}\left(r \cdot \vartheta_{a}, g_{\vartheta_{a}}, r \cdot \mathcal{A}\right)$ for all $r>0, a \in \mathcal{A} .^{11}$

The rough idea is to "shift-and-scale": we shift and scale the polytope $\mathcal{A}$, so that it is sufficiently close to a given exact one-form $d H$ in the operator norm $\|\cdot\|$ coming from $g$. Then, one can perturb the scaled section by means of Proposition 2.13 and scale back. This will be the desired section $\vartheta$ on $\mathcal{A}$. By construction, we will then see that the three bullet points are satisfied. The choices involved (i.e., choice of section $\theta$, reference pair $(H, g)$, and perturbation coming from Theorem 2.14) will prove harmless - they result in chain homotopy equivalent complexes. This is proven in the next subsection (cf. Theorem 2.19).

At the cost of imposing a smallness condition on the underlying section, we get the same results for perturbations associated with non-exact reference pairs (cf. Corollary 2.17) and the same independence of auxiliary data holds (cf. Theorem 2.22).

Proof of Theorem 2.14. As a first candidate for $\vartheta$, we pick

$$
\vartheta: \mathcal{A} \longrightarrow \Omega^{1}(M), \quad \vartheta_{a}:=\theta_{a}+d H .
$$

\footnotetext{
${ }^{11}$ Note that here the metric is not scaled.
} 
This is still a section, but does not satisfy the bullet points above. Since $\theta$ is smooth, there exists

$$
\varepsilon=\varepsilon(\theta, H, g, \delta)>0
$$

such that

$$
\varepsilon \cdot \theta_{a}+d H \text { is } \frac{C_{H}}{D \cdot 1000} \text {-close to } d H
$$

with respect to $\|\cdot\|$ induced by $g, C_{H}=C_{H}(\delta, g)>0$ and $D=D(N, g)>0$ chosen as in Proposition 2.13. Now, we can apply Proposition 2.13 to the section $\varepsilon \cdot a \mapsto \varepsilon \cdot \theta_{a}+d H$ and obtain a new section ${ }^{12}$

$$
\vartheta^{\varepsilon}: \varepsilon \cdot \mathcal{A} \longrightarrow \Omega^{1}(M) .^{12} \text {. }
$$

Finally, we scale back and redefine

$$
\vartheta=\vartheta(\theta, H, g, \varepsilon): \mathcal{A} \longrightarrow \Omega^{1}(M), \quad a \mapsto \frac{1}{\varepsilon} \cdot \vartheta^{\varepsilon}(\varepsilon \cdot a) .
$$

Thus, we have

$$
\varepsilon \cdot \vartheta_{a}=\vartheta^{\varepsilon}(\varepsilon \cdot a), \quad \forall a \in \mathcal{A} .
$$

For each $\vartheta^{\varepsilon}(\varepsilon \cdot a)$, we choose a Riemannian metric denoted by $g_{a}$ as in Proposition 2.13. Thus, $\left(\vartheta^{\varepsilon}(\varepsilon \cdot a), g_{a}\right)$ is Morse-Smale, and so is $\left(\vartheta_{a}, g_{a}\right)$, since scaling does not affect the Morse-Smale property. This proves the first bullet point.

Claim. $\mathrm{CN}_{\bullet}\left(\vartheta^{\varepsilon}(\varepsilon \cdot a), \varepsilon \cdot \mathcal{A}\right)=\mathrm{CN}_{\bullet}\left(\varepsilon \cdot \vartheta_{a}, \varepsilon \cdot \mathcal{A}\right)$ is a well-defined chain complex for any $a \in \mathcal{A}$.

Indeed, assume for contradiction that there exists a Novikov chain $\xi=$ $\sum_{\tilde{x}} \xi_{\tilde{x}} \tilde{x} \in \mathrm{CN} \bullet\left(\vartheta^{\varepsilon}(\varepsilon \cdot a), \varepsilon \cdot \mathcal{A}\right)$, such that

$$
\partial \xi \notin \mathrm{CN}_{\bullet-1}\left(\vartheta^{\varepsilon}(\varepsilon \cdot a), \varepsilon \cdot \mathcal{A}\right) \text {. }
$$

This means that there are some $\varepsilon \cdot b \in \varepsilon \cdot \mathcal{A}, c \in \mathbb{R}$ and sequences $\tilde{x}_{n}$ with $\xi_{\tilde{x}_{n}} \neq 0, \tilde{y}_{n}$ pairwise distinct, $\tilde{\gamma}_{n} \in \underline{\mathcal{M}}\left(\tilde{x}_{n}, \tilde{y}_{n} ; \tilde{f}_{\varepsilon \cdot \vartheta_{a}}\right)$, and

$$
\tilde{f}_{\vartheta^{\varepsilon}(\varepsilon \cdot b)}\left(\tilde{y}_{n}\right)=\tilde{f}_{\varepsilon \cdot \vartheta_{b}}\left(\tilde{y}_{n}\right) \geq c
$$

see Remark 2.5. Denote by $\gamma_{n}=\pi \circ \tilde{\gamma}_{n}$ the Novikov-Morse trajectories downstairs. The energy expression can then be massaged as follows:

$$
\begin{aligned}
0 \leq E\left(\tilde{\gamma}_{n}\right)=E\left(\gamma_{n}\right) & =-\int_{\gamma_{n}} \vartheta^{\varepsilon}(\varepsilon \cdot a) \\
& =-\int_{\gamma_{n}} \vartheta^{\varepsilon}(\varepsilon \cdot b)+\int_{\gamma_{n}} \vartheta^{\varepsilon}(\varepsilon \cdot b)-\vartheta^{\varepsilon}(\varepsilon \cdot a) \\
& =\tilde{f}_{\vartheta^{\varepsilon}(\varepsilon \cdot b)}\left(\tilde{x}_{n}\right)-\tilde{f}_{\vartheta^{\varepsilon}(\varepsilon \cdot b)}\left(\tilde{y}_{n}\right)+\int_{\gamma_{n}} \vartheta^{\varepsilon}(\varepsilon \cdot b)-\vartheta^{\varepsilon}(\varepsilon \cdot a)
\end{aligned}
$$

${ }^{12}$ Explicitly, this section is of the form

$$
\varepsilon \cdot a \mapsto \varepsilon \cdot \theta_{a}+d H-\sum_{i} d\left(f_{i} \cdot h_{i}\right),
$$

where $f_{i}$ depends smoothly on $\theta_{a}$ and satisfies $d f_{i}=\varepsilon \cdot \theta_{a}, f_{i}\left(x_{i}\right)=0$ around critical points $x_{i}$ of $H$, see Lemma 2.12, (5) and (6) applied to $\varepsilon \cdot \theta_{a}+d H$ and $\rho=d H$. 


$$
\leq \tilde{f}_{\vartheta^{\varepsilon}(\varepsilon \cdot b)}\left(\tilde{x}_{n}\right)-c+\int_{\gamma_{n}} \vartheta^{\varepsilon}(\varepsilon \cdot b)-\vartheta^{\varepsilon}(\varepsilon \cdot a) .
$$

Showing that the rightmost term is bounded by $m \cdot E\left(\gamma_{n}\right), m \in(0,1)$ suffices to obtain a contradiction: admitting such a bound leads to

$$
0 \leq E\left(\gamma_{n}\right) \leq(1-m)^{-1} \cdot\left(\tilde{f}_{\vartheta \varepsilon}(\varepsilon \cdot b)\left(\tilde{x}_{n}\right)-c\right) .
$$

In particular, $c \leq \tilde{f}_{\vartheta^{\varepsilon}(\varepsilon \cdot b)}\left(\tilde{x}_{n}\right)$ for all $n$. However, $\xi$ belongs to $\mathrm{CN}_{\bullet}\left(\varepsilon \cdot \vartheta_{a}, \varepsilon \cdot \mathcal{A}\right)$ and $\xi_{\tilde{x}_{n}} \neq 0$, and thus, the multi-finiteness condition implies that there are only finitely many distinct $\tilde{x}_{n}$. Up to passing to a subsequence, we can therefore assume $\tilde{x}_{n}=\tilde{x}$ and also $\tilde{y}_{n} \in \pi^{-1}(y) .{ }^{13}$ The corresponding NovikovMorse trajectories

$$
\gamma_{n} \in \underline{\mathcal{M}}\left(x, y ; \vartheta^{\varepsilon}(\varepsilon \cdot a)\right)
$$

have uniformly bounded energy

$$
E\left(\gamma_{n}\right) \leq(1-m)^{-1} \cdot\left(\tilde{f}_{\vartheta^{\varepsilon}(\varepsilon \cdot b)}(\tilde{x})-c\right) ;
$$

therefore, $\gamma_{n}$ has a $C_{\text {loc }}^{\infty}$-convergent subsequence. At the same time $\underline{\mathcal{M}}\left(x, y ; \vartheta^{\varepsilon}(\varepsilon \cdot a)\right)$ is a 0 -dimensional manifold, which means that the convergent subsequence $\gamma_{n}$ eventually does not depend on $n$. This contradicts our assumption that the endpoints $\tilde{y}_{n}$ upstairs are pairwise disjoint.

Therefore, we are only left to show the bound

$$
A\left(\gamma_{n}\right):=\int_{\gamma_{n}} \vartheta^{\varepsilon}(\varepsilon \cdot b)-\vartheta^{\varepsilon}(\varepsilon \cdot a) \leq \frac{1}{2} E\left(\gamma_{n}\right)
$$

to conclude the Claim. For this purpose, we define

$$
\mathcal{S}_{n}:=\left\{s \in \mathbb{R} \mid\left\|\nabla^{g_{a}}\left(\vartheta^{\varepsilon}(\varepsilon \cdot a)\right)\left(\gamma_{n}(s)\right)\right\|_{g_{a}} \geq \frac{C_{H}}{8}\right\} .
$$

The crucial observation is that both $\vartheta^{\varepsilon}(\varepsilon \cdot b)$ and $\vartheta^{\varepsilon}(\varepsilon \cdot a)$ agree with $d H$ around Crit $(H)$, by choice of $\vartheta^{\varepsilon}$ via Proposition 2.13. In particular $\vartheta^{\varepsilon}(\varepsilon \cdot b)-\left.\vartheta^{\varepsilon}(\varepsilon \cdot a)\right|_{B_{\delta}(z)}=0, \quad \forall z \in \operatorname{Crit}(H)=Z\left(\vartheta^{\varepsilon}(\varepsilon \cdot b)\right)=Z\left(\vartheta^{\varepsilon}(\varepsilon \cdot a)\right)$.

Lemma 2.12 says that for $s \in \mathbb{R} \backslash \mathcal{S}_{n}$, we get

$$
\gamma_{n}(s) \in \bigcup_{z \in Z\left(\vartheta^{\varepsilon}(\varepsilon \cdot a)\right)} B_{\delta}(z)
$$

consequently

$$
\int_{\mathbb{R} \backslash \mathcal{S}_{n}}\left(\vartheta^{\varepsilon}(\varepsilon \cdot b)-\vartheta^{\varepsilon}(\varepsilon \cdot a)\right) \dot{\gamma}_{n}(s) \mathrm{d} s=0 .
$$

The Lebesgue measure $\mu\left(\mathcal{S}_{n}\right)$ can be bounded using the energy

$E\left(\gamma_{n}\right)=-\int_{\gamma_{n}} \vartheta^{\varepsilon}(\varepsilon \cdot a)=\int_{\mathbb{R}}\left\|\nabla^{g_{a}}\left(\vartheta^{\varepsilon}(\varepsilon \cdot a)\right)\left(\gamma_{n}(s)\right)\right\|_{g_{a}}^{2} \mathrm{~d} s \geq \mu\left(\mathcal{S}_{n}\right) \cdot\left(\frac{C_{H}}{8}\right)^{2} ;$

${ }^{13}$ The latter is possible, since $Z\left(\varepsilon \cdot \vartheta_{a}\right)=Z(\varepsilon \cdot d H)=\operatorname{Crit}(H)$ is finite. 
thus

$$
\mu\left(\mathcal{S}_{n}\right) \leq\left(\frac{8}{C_{H}}\right)^{2} \cdot E\left(\gamma_{n}\right)
$$

And finally

$$
\begin{aligned}
\left|A\left(\gamma_{n}\right)\right| \leq & \left\|\vartheta^{\varepsilon}(\varepsilon \cdot b)-\vartheta^{\varepsilon}(\varepsilon \cdot a)\right\|_{g_{a}} \cdot \int_{\mathcal{S}_{n}}\left\|\dot{\gamma}_{n}(s)\right\|_{g_{a}} \mathrm{~d} s \quad \text { by }(8), \\
\leq & \left(\left\|\vartheta^{\varepsilon}(\varepsilon \cdot b)-d H\right\|_{g_{a}}\right. \\
& \left.+\left\|d H-\vartheta^{\varepsilon}(\varepsilon \cdot a)\right\|_{g_{a}}\right) \cdot \mu\left(\mathcal{S}_{n}\right)^{\frac{1}{2}} \cdot E\left(\gamma_{n}\right)^{\frac{1}{2}} \\
\leq & 5 D \cdot\left(\left\|\varepsilon \cdot \vartheta_{b}-d H\right\|\right. \\
& \left.+\left\|d H-\varepsilon \cdot \vartheta_{a}\right\|\right) \cdot \frac{8}{C_{H}} \cdot E\left(\gamma_{n}\right) \quad \text { Proposition } 2.13,(9), \\
\leq & \frac{80 \cdot D \cdot C_{H}}{D \cdot 1000 \cdot C_{H}} \cdot E\left(\gamma_{n}\right) \quad \text { by choice of scaling } \varepsilon>0, \\
< & \frac{1}{10} E\left(\gamma_{n}\right) .
\end{aligned}
$$

This proves the Claim.

Now, we observe that scaling $\vartheta_{a}$ by $r>0$ does not affect the zeros and that the moduli spaces associated with $\left(\vartheta_{a}, g_{a}\right)$ are in one-to-one correspondence with those of $\left(r \cdot \vartheta_{a}, g_{a}\right)$. It is also clear that the multi- finiteness condition imposed by $\mathcal{A}$ is equivalent to that of $r \cdot \mathcal{A}$. All in all, this means that for any $r>0$, the polytope chain complexes associated with $\left(r \cdot \vartheta_{a}, g_{a}\right)$ agree with each other. This proves the ray invariance. Setting $r=\frac{1}{\varepsilon}$ and using the Claim prove the remaining first bullet point.

Remark 2.15. Instead of running the argument for the sections $\vartheta^{\varepsilon}: \varepsilon \cdot \mathcal{A} \rightarrow$ $\Omega^{1}(M)$, we could also work with $\vartheta=\frac{1}{\varepsilon} \cdot \vartheta^{\varepsilon}: \mathcal{A} \rightarrow \Omega^{1}(M)$ by directly by applying Proposition 2.13 to the section

$$
a \mapsto \theta_{a}+d\left(\varepsilon^{-1} H\right)
$$

and $\left(\varepsilon^{-1} H, g\right)$. These two approaches are equivalent; the only difference is psychological: we find it more natural to visualize the shrinking of the polytope opposed to the scaling of Morse functions. Note that the analogous bound at the end of the proof of Theorem 2.14 holds upon replacing $H$ by $\varepsilon^{-1} H$. This follows from the nice scaling behavior of the PS constants:

$$
C_{\varepsilon^{-1} H}(\delta, g)=\varepsilon^{-1} C_{H}(\delta, g) ;
$$

therefore, " $\varepsilon \cdot \theta_{a}+d H$ is $\frac{C_{H}}{D \cdot 1000}$-close to $d H$ " if and only if " $\theta_{a}+d\left(\varepsilon^{-1} H\right)$ is $\frac{C_{\varepsilon}-1_{H}}{D \cdot 1000}=\frac{C_{H}}{\varepsilon \cdot D \cdot 1000}$-close to $d\left(\varepsilon^{-1} H\right)$ ".

Remark 2.16. The skeptical reader might wonder whether $\partial_{\vartheta_{a}}^{2}=0$ really holds. Viewing the chain group $\mathrm{CN}_{\bullet}\left(\vartheta_{a}, \mathcal{A}\right)$ as a certain twisted chain group allows for a quick and simple proof-see Remark 2.35.

The key in the proof of Theorem 2.14 was to obtain control over the energy by perturbing the section $\theta: \mathcal{A} \rightarrow \Omega^{1}(M)$ via Proposition 2.13. The 
perturbation was chosen, so that there would be no contribution to the energy near the zeros. Similar ideas to control the energy can be found in $[2$, Subsection 3.6.2], [25].

The question remains why we used an (exact) reference pair $(H, g)$ instead of a more general Morse-Smale pair $(\rho, g)$ in Theorem 2.14. The answer is simple: the given argument already breaks down in the very first line - the corresponding $\vartheta$ is not a section anymore, since the $\rho$-shift changes the cohomology class. However, whenever the section $\theta: \mathcal{A} \rightarrow \Omega^{1}(M)$ is already sufficiently close to $(\rho, g)$ in terms of the corresponding PS-constant $C_{\rho}>0$, we do not need to shift and scale $\theta$, and can perturb $\theta$ directly:

Corollary 2.17. Let $(\rho, g)$ be a Morse-Smale pair, $C=C_{\rho}>0, N=\bigcup_{i} B_{2 \delta}\left(x_{i}\right)$ with $x_{i} \in Z(\rho)$, and $D=d(N, \delta)>0$ as in Proposition 2.13. Let $\theta: \mathcal{A} \rightarrow$ $\Omega^{1}(M)$ be a smooth section, such that

$$
\left\|\theta_{a}-\rho\right\|<\frac{C_{\rho}}{D \cdot 1000},
$$

with $\|\cdot\|$ the operator norm induced by $g$. Then, there exists a perturbed section

$$
\vartheta=\vartheta(\theta, \rho, g): \mathcal{A} \longrightarrow \Omega^{1}(M),
$$

and $g_{\vartheta_{a}}$, such that the same conclusions as in Theorem 2.14 hold.

Proof. Upon replacing $\varepsilon \cdot \theta_{a}+d H$ and $d H$ with $\theta_{a}$ and $\rho$, the proof is word for word the same as the one of Theorem 2.14.

\subsection{Independence of the data}

The section $\vartheta=\vartheta(\theta, H, g)$ constructed in Theorem 2.14 does not only depend on $(\theta, H, g)$, but also comes with a choice of scaling $\varepsilon(\theta, H, g, \delta)>0$. We shall prove that any valid perturbation $\vartheta^{i}=\vartheta^{i}\left(\theta_{i}, H_{i}, g_{i}, \varepsilon_{i}\right)$ in the sense of Theorem 2.14 gives rise to chain homotopy equivalent chain complexes. The same is true for perturbations coming from Corollary 2.17, and at the end of the subsection, we will show that both perturbations lead to chain homotopy equivalent Novikov complexes.

Remark 2.18. All the chain maps and chain homotopy equivalences constructed from here on are Novikov-module morphisms, i.e., linear over the Novikov ring. We will not explicitly state this every time for better readability.

Theorem 2.19. For $i=0,1$, let $\theta_{i}: \mathcal{A} \rightarrow \Omega^{1}(M)$ be sections, $\left(H_{i}, g_{i}\right)$ MorseSmale pairs and $\delta_{i}>0, \varepsilon_{i}\left(\theta_{i}, H_{i}, g_{i}, \delta_{i}\right)>0$ as in proof of Theorem 2.14.

Then any two perturbed sections

$$
\vartheta^{i}=\vartheta^{i}\left(\theta_{i}, H_{i}, g_{i}, \varepsilon_{i}\right): \mathcal{A} \rightarrow \Omega^{1}(M),
$$

in the sense of Theorem 2.14, induce chain homotopy equivalent polytope complexes

$$
\mathrm{CN}_{\bullet}\left(\vartheta_{a}^{0}, \mathcal{A}\right) \simeq \mathrm{CN}_{\bullet}\left(\vartheta_{a}^{1}, \mathcal{A}\right), \quad \forall a \in \mathcal{A}
$$


Proof of Theorem 2.19. We may assume $\varepsilon_{1} \geq \varepsilon_{0}$. Denote by

$$
\vartheta^{i}\left(\sigma_{i}, H_{i}, g_{i}, \varepsilon_{i}\right), \quad i=0,1,
$$

the respective sections on $\mathcal{A}$ as in the first part of the proof of Theorem 2.14. Let

$$
h: \mathbb{R} \rightarrow \mathbb{R}_{\geq 0}
$$

be a positive smooth function with $h \equiv 0$ on $(-\infty, e)$ and $h \equiv 1$ on $(1-$ $e,+\infty)$, for some small $e>0$, and set

$$
\vartheta^{s}=(1-h(s)) \cdot \vartheta^{0}+h(s) \cdot \vartheta^{1} .
$$

Fix $a \in \mathcal{A}$ and pick $g_{i}:=g_{\vartheta_{a}^{i}}, i=0,1$ two metrics as in Theorem 2.14. Let $g_{s}=g_{s}(a)$ be a homotopy of Riemannian metrics connecting $g_{0}$ to $g_{1}$ and assume that $\left(\vartheta_{a}^{s}, g_{s}\right)$ is regular-this is rectified by Remark 2.20. Note that here $g_{s}$ actually depends on $a$.

To this regular homotopy, we can now associate a chain continuation

$$
\Psi^{10}: \mathrm{CN} \bullet\left(\vartheta_{a}^{0}, \mathcal{A}\right) \longrightarrow \mathrm{CN} \bullet\left(\vartheta_{a}^{1}, \mathcal{A}\right), \quad \xi=\sum_{\tilde{x}} \xi_{\tilde{x}} \tilde{x} \mapsto \sum_{\tilde{x}, \tilde{y}} \xi_{\tilde{x}} \cdot \# \text { alg } \mathcal{M}\left(\tilde{x}, \tilde{y} ; \tilde{f}_{\vartheta_{a}^{s}}\right) \tilde{y}
$$

Analogously to the case of the boundary operator in Theorem 2.14, proving that $\Psi^{10}$ defines a well-defined Novikov chain map essentially boils down to proving that it respects the multi-finiteness condition - the rest follows by standard Novikov-Morse techniques. Thus, proceeding as in Theorem 2.14 reveals that it suffices ${ }^{14}$ to bound

$$
\int_{\gamma_{n}} \vartheta_{b}^{s}-\vartheta_{a}^{s}
$$

by either a multiple $m \in(0,1)$ of the energy $E\left(\gamma_{n}\right)$, where $b$ is some cohomology class in $\mathcal{A}$, or a uniform bound ${ }^{15}$ altogether. Set

$$
\begin{aligned}
& \mathcal{S}_{n}^{0}:=\left\{s \in(-\infty, 0) \mid\left\|\nabla^{g_{0}} \vartheta_{a}^{0}\left(\gamma_{n}(s)\right)\right\|_{g_{0}} \geq \frac{C_{H}}{\varepsilon_{0} \cdot 8}\right\}, \\
& \mathcal{S}_{n}^{1}:=\left\{s \in(1,+\infty) \mid\left\|\nabla^{g_{1}} \vartheta_{a}^{1}\left(\gamma_{n}(s)\right)\right\|_{g_{1}} \geq \frac{C_{H}}{\varepsilon_{1} \cdot 8}\right\} .
\end{aligned}
$$

This time around we need to divide by $\varepsilon_{i}$ as we are running the continuation directly on the original polytope $\mathcal{A}$ instead of the scaled polytope (see Remark 2.15). As in the previous proof of Theorem 2.14, the $s \in \mathbb{R}_{\leq 0} \backslash \mathcal{S}_{n}^{0}$ and

${ }^{14}$ This is also implicitly using that $\tilde{f}_{\vartheta_{b}^{s}}=\tilde{f}_{\vartheta_{b}^{0}}+h_{s} \circ \pi$ for a smooth family $h_{s} \in C^{\infty}(M)$, since $\vartheta_{b}^{s}$ are cohomologous for all $s$. Hence

$$
-\int_{\gamma_{n}} \vartheta_{b}^{s}=-\tilde{f}_{\vartheta_{b}^{1}}\left(\tilde{y}_{n}\right)+\tilde{f}_{\vartheta_{b}^{0}}\left(\tilde{x}_{n}\right)+\underbrace{\int_{[0,1]} \frac{\partial h_{s}}{\partial s}\left(\gamma_{n}(s)\right) \mathrm{d} s}_{\leq C},
$$

for some uniform constant $C$.

${ }^{15}$ Uniform in $b$ and $n \in \mathbb{N}$, that is. 
$s \in \mathbb{R}_{\geq 1} \backslash \mathcal{S}_{n}^{1}$ do not contribute to $(15)$ as $\gamma_{n}(s)$ will be near the zeros of $H_{i}$, where $\vartheta_{b}^{i}=\vartheta_{a}^{i}$. On the other hand, using similar arguments, we obtain

$$
\begin{aligned}
\left|\int_{\mathcal{S}_{n}^{0} \cup \mathcal{S}_{n}^{1}}\left(\vartheta_{b}^{s}-\vartheta_{a}^{s}\right) \dot{\gamma}_{n}(s) \mathrm{d} s\right| & \leq 2 \cdot \max _{i=0,1}\left\|\vartheta_{b}^{i}-\vartheta_{a}^{i}\right\|_{i} \cdot \mu\left(\mathcal{S}_{n}^{i}\right)^{\frac{1}{2}} \cdot E\left(\gamma_{n}\right)^{\frac{1}{2}} \\
& \leq 2 \cdot \max _{i=0,1}\left(\left\|\vartheta_{b}^{i}-d H_{i}\right\|_{i}+\left\|d H_{i}-\vartheta_{a}^{i}\right\|_{i}\right) \cdot \frac{\varepsilon_{i} \cdot 8}{C_{H}} \cdot E\left(\gamma_{n}\right) \\
& \leq \max _{i=0,1} \frac{4 \cdot 5 \cdot D \cdot \varepsilon_{i} \cdot 8 \cdot C_{H}}{D \cdot \varepsilon_{i} \cdot 1000 \cdot C_{H}} \cdot E\left(\gamma_{n}\right) \\
& \leq \frac{1}{5} \cdot E\left(\gamma_{n}\right),
\end{aligned}
$$

where we have used Proposition 2.13 as in Theorem 2.14. We are left to bound (15) for $s \in[0,1]$. For this, we compute via Cauchy-Schwarz

$$
\left|\int_{[0,1]}\left(\vartheta_{b}^{s}-\vartheta_{a}^{s}\right) \dot{\gamma}_{n}(s) \mathrm{d} s\right| \leq \max _{s \in[0,1]}\left\|\vartheta_{b}^{s}-\vartheta_{a}^{s}\right\|_{s} \cdot E\left(\gamma_{n}\right)^{\frac{1}{2}} .
$$

By compactness of $[0,1], \mathcal{A}$ and continuity of $\vartheta:[0,1] \times \mathcal{A} \rightarrow \Omega^{1}(M)$, we may bound

$$
\max _{s \in[0,1]}\left\|\vartheta_{b}^{s}-\vartheta_{a}^{s}\right\|_{s} \leq F
$$

where $F>0$ is a uniform constant in $s \in[0,1]$ and $b \in \mathcal{A}$-recall that $g_{s}$ depends on $a$, but that does not matter. In particular, this proves

$$
\left|\int_{\gamma_{n}} \vartheta_{b}^{s}-\vartheta_{a}^{s}\right| \leq F \cdot E\left(\gamma_{n}\right)^{\frac{1}{2}}+\frac{1}{5} \cdot E\left(\gamma_{n}\right), \quad \forall n \in \mathbb{N}, b \in \mathcal{A} .
$$

A case distinction now does the job: for any $n \in \mathbb{N}$, we either have $\frac{1}{5} E\left(\gamma_{n}\right) \geq$ $F \cdot E\left(\gamma_{n}\right)^{\frac{1}{2}}$ or $\frac{1}{5} E\left(\gamma_{n}\right)<F \cdot E\left(\gamma_{n}\right)^{\frac{1}{2}}$. In the first case, we can bound the norm of (15) by $\frac{2}{5} \cdot E\left(\gamma_{n}\right)$, whereas in the second case, we get $\frac{1}{5} \cdot E\left(\gamma_{n}\right)^{\frac{1}{2}}<F$, and thus, we may bound the norm of (15) by $10 F^{2}$. This proves

$$
\left|\int_{\gamma_{n}} \vartheta_{b}^{s}-\vartheta_{a}^{s}\right| \leq \max \left\{10 F^{2}, \frac{2}{5} E\left(\gamma_{n}\right)\right\} .
$$

As explained before, this suffices to conclude that $\Psi^{10}$ defines a well-defined Novikov chain map, which defines the desired chain homotopy equivalence (see proof of Theorem 2.14 for more details). This concludes the proof.

Remark 2.20. The (linear) homotopy $\left(\vartheta_{a}^{s}, g_{s}\right)$ chosen in the proof of Theorem 2.19 might be non-regular. One can replace $\left(\vartheta_{a}^{s}, g_{s}\right)$ with an arbitrarily close regular homotopy $\left(\left(\vartheta_{a}^{s}\right)^{\prime}, g_{s}^{\prime}\right)$ connecting the same data. The only bit where this affects the previous argument in Theorem 2.19 is when trying to bound $\max _{s \in[0,1]}\left\|\vartheta_{b}^{s}-\left(\vartheta_{a}^{s}\right)^{\prime}\right\|_{s}^{\prime}$. Using that $\vartheta_{a}^{s}$ is smooth in $s$ and close to $\left(\vartheta_{a}^{s}\right)^{\prime}$, we still get the desired uniform bound $b$.

As a consequence of Theorems 2.14 and 2.19, we obtain the analogue results for restrictions to subpolytopes $\mathcal{B} \subseteq \mathcal{A}$ : 
Corollary 2.21. Let $\theta: \mathcal{A} \rightarrow \Omega^{1}(M)$ be a section. Then, for every perturbed section $\vartheta: \mathcal{A} \rightarrow \Omega^{1}(M)$ coming from Theorem 2.14 and subpolytope $\mathcal{B} \subseteq \mathcal{A}$, we obtain a well- defined polytope chain complex

$$
\left(\mathrm{CN}_{\bullet}\left(\vartheta_{a},\left.\mathcal{A}\right|_{\mathcal{B}}\right), \partial_{\vartheta_{a}}\right), \quad \forall a \in \mathcal{A},
$$

satisfying all the bullet points of Theorem 2.14. Any other choice $\vartheta^{\prime}=\vartheta^{\prime}$ $\left(\theta^{\prime}, H^{\prime}, g^{\prime}\right)$ does not affect the chain complexes up to chain homotopy equivalence.

Moreover, the inclusion

$$
\iota_{\mathcal{B}}: \mathrm{CN}_{\bullet}\left(\vartheta_{a}, \mathcal{A}\right) \longrightarrow \mathrm{CN}_{\bullet}\left(\vartheta_{a},\left.\mathcal{A}\right|_{\mathcal{B}}\right)
$$

defines a Novikov-linear chain map for all $a \in \mathcal{A}$.

Proof. The proof of the first part is literally the same as in Theorems 2.14 and 2.19. To see that the inclusion defines a chain map, it suffices to observe that both boundary operators in (17) are identical upon restricting to the smaller complex $\mathrm{CN}_{\bullet}\left(\vartheta_{a}, \mathcal{A}\right)$.

In the preceding subsection, we also defined a polytope chain complex variant using perturbed sections with respect to non-exact reference pairs (cf. Corollary 2.17). While this variant requires the underlying section to satisfy some a priori smallness conditions, it does agree with the polytope chain complex variant of Theorem 2.14.

Theorem 2.22. Let $(\rho, g)$ and $\theta: \mathcal{A} \rightarrow \Omega^{1}(M)$ be as in Corollary 2.17, and denote by $\vartheta^{\rho}=\vartheta(\theta, \rho, g)$ the corresponding perturbed section. Let $\vartheta^{H}=$ $\vartheta\left(\theta, H, g_{H}, \varepsilon\right)$ be any perturbed section as in Theorem 2.14. Then,

$$
\mathrm{CN}_{\bullet}\left(\vartheta_{a}^{\rho}, \mathcal{A}\right) \simeq \mathrm{CN}_{\bullet}\left(\vartheta_{a}^{H}, \mathcal{A}\right), \quad \forall a \in \mathcal{A} .
$$

Let $\theta_{i}: \mathcal{A} \rightarrow \Omega^{1}(M)$ be any other two sections $i=0,1$ with reference pairs $\left(\rho_{i}, g_{i}\right)$ satisfying the conditions of Corollary 2.17. Then, for any two choices $\vartheta^{\rho_{i}}=\vartheta\left(\theta_{i}, \rho_{i}, g_{i}\right)$, one has

$$
\mathrm{CN}_{\bullet}\left(\vartheta_{a}^{\rho_{0}}, \mathcal{A}\right) \simeq \mathrm{CN}_{\bullet}\left(\vartheta_{a}^{\rho_{1}}, \mathcal{A}\right), \quad \forall a \in \mathcal{A} .
$$

Moreover, both (18) and (19) continue to hold in the restricted case $\mathcal{B} \subseteq \mathcal{A}$.

Proof. The proof idea is again arguing via continuations as in Theorem 2.19 above - we will use the latter as carbon copy and adapt the same notation. Define

$$
\vartheta^{s}=(1-h(s)) \cdot \vartheta^{\rho}+h(s) \cdot \vartheta^{H} .
$$

By the same logic as in Theorem 2.19, it suffices to control the expression

$$
\int_{\gamma} \vartheta_{b}^{s}-\vartheta_{a}^{s}
$$

for all $b \in \mathcal{A}$, to get the desired continuation chain map to conclude (18). For this purpose, we define

$$
\mathcal{S}^{\rho}:=\left\{s \in(-\infty, 0) \mid\left\|\nabla^{g_{0}} \vartheta_{a}^{\rho}(\gamma(s))\right\|_{g_{0}} \geq \frac{C_{\rho}}{8}\right\},
$$




$$
\mathcal{S}^{H}:=\left\{s \in(1,+\infty) \mid\left\|\nabla^{g_{1}} \vartheta^{H} a(\gamma(s))\right\|_{g_{1}} \geq \frac{C_{H}}{\varepsilon \cdot 8}\right\} .
$$

Here, $g_{0}$ and $g_{1}$ (abusively) denote Riemannian metrics $g_{\vartheta_{a}^{\rho}}$ and $g_{\vartheta_{a}^{H}}$ coming from Proposition 2.13.

Observe that by assumption and choice of $\left(\vartheta_{a}^{\rho}, g_{0}\right)$, we have

$$
\left\|\vartheta_{b}^{\rho}-\rho\right\|_{g_{0}} \leq 5 \cdot D_{0} \cdot\left\|\theta_{b}-\rho\right\|_{g} \leq \frac{5 \cdot D_{0} \cdot C_{\rho}}{D_{0} \cdot 1000}
$$

see proof of Corollary 2.17 and Proposition 2.13.

Just as in the proof of Theorem 2.19, there is no contribution to (20) for $s$ in the complement of $[0,1] \cup \mathcal{S}^{\rho} \cup \mathcal{S}^{H}$. At the same time, we can again bound

$$
\left|\int_{\mathcal{S}^{\alpha}}\left(\vartheta_{b}^{s}-\vartheta_{a}^{s}\right) \dot{\gamma}(s) \mathrm{d} s\right|<\frac{1}{10} \cdot E(\gamma)
$$

and

$$
\left|\int_{[0,1]}\left(\vartheta_{b}^{s}-\vartheta_{a}^{s}\right) \dot{\gamma}(s) \mathrm{d} s\right| \leq F \cdot E(\gamma)^{\frac{1}{2}} .
$$

This suffices to obtain the desired control over (20) and proves (18); see proof of Theorem 2.19 for more details. Last but not least, (19) follows by applying (18) twice:

$$
\mathrm{CN}_{\bullet}\left(\vartheta_{a}^{\rho_{0}}, \mathcal{A}\right) \simeq \mathrm{CN}_{\bullet}\left(\vartheta_{a}^{H}, \mathcal{A}\right) \simeq \mathrm{CN}_{\bullet}\left(\vartheta_{a}^{\rho_{1}}, \mathcal{A}\right), \quad \forall a \in \mathcal{A}
$$

Theorems 2.19 and 2.22 readily imply:

Corollary 2.23. Let $\theta_{i}: \mathcal{A} \rightarrow \Omega^{1}(M)$ with $i=0,1$ be two sections and $\vartheta^{i}$ associated perturbations as in Theorem 2.14 (or Corollary 2.17). Then, the resulting polytope Novikov homologies are isomorphic

$$
\mathrm{HN}_{\bullet}\left(\vartheta_{a}^{0}, \mathcal{A}\right) \cong \mathrm{HN}_{\bullet}\left(\vartheta_{a}^{1}, \mathcal{A}\right), \quad \forall a \in \mathcal{A} \text {. }
$$

Corollary 2.23 is the analogue to the independence of Morse-Smale pairs $(\alpha, g)$ in the case of ordinary Novikov homology. The latter can also be recovered from the former by taking the trivial polytope $\mathcal{A}=\langle a\rangle$. Nevertheless, keeping track of the section $\theta$, or rather its perturbations, will prove useful, especially when establishing the commutative diagram in the Main Theorem 2.24 .

\subsection{Non-exact deformations and proof of the main theorem}

The power of the polytope machinery will become evident in this subsectionroughly speaking, the notion of polytopes allows us to compare the Novikov homologies coming from two different cohomology classes, see Main Theorem 2.24. In Sect. 3, we present some applications of the Main Theorem 2.24.

Theorem 2.24. (Main Theorem) Let $\mathcal{A} \subset H_{\mathrm{dR}}^{1}(M)$ be a polytope and $\theta: \mathcal{A} \rightarrow$ $\Omega^{1}(M)$ a section. Then, there exists a perturbation $\vartheta: \mathcal{A} \rightarrow \Omega^{1}(M)$ of $\theta$, such that for every subpolytope $\mathcal{B} \subseteq \mathcal{A}$ and any two cohomology classes $a, b \in \mathcal{A}$, there exists a commutative diagram 


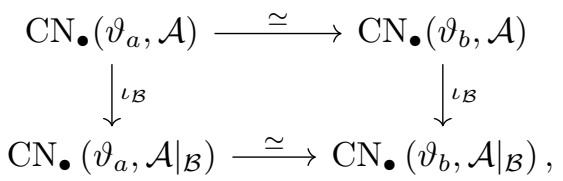

where the horizontal maps are Novikov-linear chain homotopy equivalences. In particular

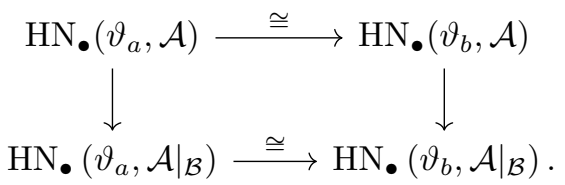

with all the maps being Novikov linear.

Proof. Most of the ideas have already been established in the previous subsection, especially in the proof of Theorems 2.14 and 2.19. Fix a reference Morse-Smale pair $(H, g)$ and pick a perturbation $\vartheta=\vartheta(\theta, H, g, \varepsilon)$ as in Theorem 2.14. Let $h:[0,1] \rightarrow \mathbb{R}$ be a smooth function as in (13) and define a homotopy

$$
\vartheta_{a b}^{s}:=(1-h(s)) \cdot \vartheta_{a}+h(s) \cdot \vartheta_{b}, \quad \forall s \in \mathbb{R} .
$$

Pick $g_{s}$ a smooth homotopy connecting the two metrics $g_{\vartheta_{a}}$ and $g_{\vartheta_{b}}$ and assume that $\left(\vartheta_{a b}^{s}, g_{s}\right)$ is regular (see Remark 2.20). The idea now is to show that the chain continuation map

$$
\Psi^{b a}: \mathrm{CN}_{\bullet}\left(\vartheta_{a}, g_{\vartheta_{a}}, \mathcal{A}\right) \longrightarrow \mathrm{CN}_{\bullet}\left(\vartheta_{b}, g_{\vartheta_{b}}, \mathcal{A}\right)
$$

associated with the regular homotopy $\left(\vartheta_{a b}^{s}, g_{s}\right)$ is well defined and makes the desired diagram commute. The argument that $\Psi^{b a}$ is a well-defined Novikov chain map is the same as in Theorem 2.19 and follows by controlling terms of the form:

$$
\int_{\gamma} \vartheta_{c}-\vartheta_{a b}^{s}, \quad \text { with } c \in \mathcal{A} .
$$

Note that this time around we do not need to put an $s$-dependence on $\vartheta_{c}$ (this corresponds to $\vartheta_{b}$ in the proof of Theorem 2.19), since the endpoints of $\vartheta_{a b}^{s}$ have the same zeros as $\vartheta_{c}$, namely $Z\left(\vartheta_{c}\right)=\operatorname{Crit}(H)$. The $s$-dependence on $\vartheta_{c}$ is the only bit that used the cohomologous assumption in Theorem 2.19 , and indeed, the remaining part of the proof is verbatim the same and is thus omitted.

We use the very same homotopy to define a chain continuation

$$
\left.\Psi^{b a}\right|_{\mathcal{B}}: \mathrm{CN}_{\bullet}\left(\vartheta_{a},\left.\mathcal{A}\right|_{\mathcal{B}}\right) \longrightarrow \mathrm{CN}_{\bullet}\left(\vartheta_{b},\left.\mathcal{A}\right|_{\mathcal{B}}\right) \text {. }
$$

From this, we obtain the following commutative diagram on the chain level:

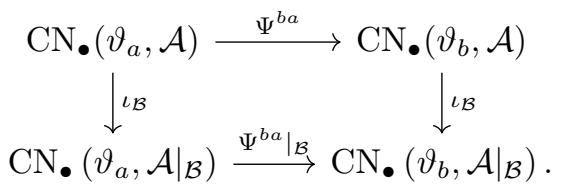


Here, the $\iota_{\mathcal{B}}$ denote the inclusions (17), which are chain maps (cf. Corollary $2.21)$. By symmetry and the standard argument, we get continuations $\Psi^{a b}$ and $\left.\Psi^{a b}\right|_{\mathcal{B}}$ in the opposite direction by reversing the underlying regular homotopy. It is also easy to see that continuation maps are linear over the underlying Novikov ring. This proves that the two horizontal chain maps above define the desired chain homotopy equivalences. In particular, the chain diagram above induces the desired diagram in homology and thus concludes the proof.

Remark 2.25. In light of Theorems 2.19, 2.22 and Corollary 2.23, one can upgrade Theorem 2.24 and use different sections on the left and on the right of the the diagram, that is

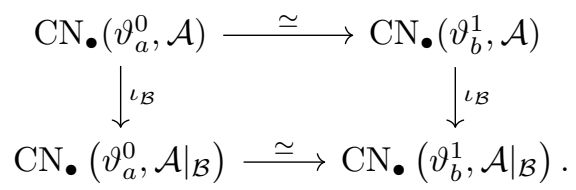

The upper and lower chain homotopy equivalences, however, do come from compositions of chain continuations rather than genuine chain continuations.

\subsection{Twisted Novikov complex}

Throughout this subsection, we shall assume that $\theta: \mathcal{A} \rightarrow \Omega^{1}(M)$ has already been perturbed as in Theorem 2.14. ${ }^{16}$ We present an alternative description of $\mathrm{CN}_{\bullet}\left(\theta_{a}, \mathcal{A}\right)$ by means of local coefficients. For an extensive treatment of local coefficients, we recommend [23] and [1, Chapter 2] in the case of Morse homology.

Definition 2.26. Let $G \subseteq \mathbb{R}$ be an additive subgroup. Then, define

$$
\operatorname{Nov}(G ; \mathbb{Z}):=\operatorname{Nov}(G)
$$

as the ring ${ }^{17}$ consisting of formal sums $\sum_{g \in G} n_{g} t^{g}$ with $n_{g} \in \mathbb{Z}$, satisfying the finiteness condition

$$
\forall c \in \mathbb{R}:\left\{g \mid n_{g} \neq 0, g<c\right\} \text { is finite. }
$$

Whenever $G$ is the image of a period homomorphism $\Phi_{a}: \pi_{1}(M) \rightarrow \mathbb{R}$, we write

$$
\operatorname{Nov}(a):=\operatorname{Nov}(G), \quad G=\operatorname{im}\left(\Phi_{a}\right) .
$$

It turns out that $\operatorname{Nov}(a)$ is isomorphic to $\Lambda_{a}$, where the isomorphism is given by sending a deck transformation $A \in \Gamma_{a}$ to $t^{\Phi_{a}(A)}$ - both finiteness conditions match and we obtain:

Proposition 2.27. For any cohomology class $a \in H_{\mathrm{dR}}^{1}(M)$, we have

$$
\Lambda_{a} \cong \operatorname{Nov}(a)
$$

as rings.

\footnotetext{
${ }^{16}$ Strictly speaking, we could also use perturbations coming from Corollary 2.17 at the expanse of working with small sections. For the sake of exposition, we refrain from stating this explicitly.

${ }^{17}$ The addition and multiplication of $\operatorname{Nov}(G)$ are the obvious ones: $n_{g} t^{g}+m_{g} t^{g}=\left(n_{g}+\right.$ $\left.m_{g}\right) t^{g}$ and $\left(n_{g} t^{g}\right) \cdot\left(n_{h} t^{h}\right):=n_{g} \cdot n_{h} t^{g+h}$.
} 
In view of Proposition 2.27, we will also refer to $\operatorname{Nov}(a)$ as Novikov ring of $a$. Inspired by the definition of $\operatorname{Nov}(a)$, we will now define yet another ring $\operatorname{Nov}(\mathcal{A})$, which will be isomorphic to $\Lambda_{\mathcal{A}}$ almost by definition.

Definition 2.28. Let $\mathcal{A}:=\left\langle a_{0}, \ldots, a_{k}\right\rangle$ be a polytope. Define

$$
\operatorname{Nov}(\mathcal{A} ; \mathbb{Z}):=\operatorname{Nov}(\mathcal{A}),
$$

as the ring consisting of elements

$$
\sum_{A \in \Gamma_{\mathcal{A}}} n_{A} t_{0}^{\Phi_{a_{0}}(A)} \cdots t_{k}^{\Phi_{a_{k}}(A)}, \quad n_{A} \in \mathbb{Z},
$$

with a multi-finiteness condition

$$
\forall l=0, \ldots, k, \forall c \in \mathbb{R}: \quad\left\{A \mid n_{A} \neq 0, \Phi_{a_{l}}(A)<c\right\} \text { is finite. }
$$

Similarly, for any subpolytope $\mathcal{B} \subseteq \mathcal{A}$, we define a (potentially) larger group

$$
\operatorname{Nov}\left(\left.\mathcal{A}\right|_{\mathcal{B}}\right) \supseteq \operatorname{Nov}(\mathcal{A})
$$

consisting of the same formal sums (21), but with a (potentially) less-restrictive multi- finiteness condition

For all $l$ such that $a_{l} \in \mathcal{B}$ and $c \in \mathbb{R}:\left\{A \mid n_{A} \neq 0, \Phi_{a_{l}}(A)<c\right\}$ is finite. Analogously to Proposition 2.27, we have: ${ }^{18}$

Proposition 2.29. For any polytope $\mathcal{A}$, we have

$$
\Lambda_{\mathcal{A}} \cong \operatorname{Nov}(\mathcal{A})
$$

as rings. Similarly, we obtain

$$
\Lambda_{\left.\mathcal{A}\right|_{\mathcal{B}}} \cong \operatorname{Nov}\left(\left.\mathcal{A}\right|_{\mathcal{B}}\right)
$$

as well.

Let us mention that these Novikov rings are commutative rings, since $\Gamma_{\mathcal{A}}$ is abelian. Indeed, the commutator subgroup of $\pi_{1}(M)$ is contained in every kernel $\operatorname{ker}\left(a_{l}\right)$; in particular

$$
A B A^{-1} B^{-1} \in \bigcap_{l=0}^{k} \operatorname{ker}\left(a_{l}\right), \text { thus } A B A^{-1} B^{-1}=0 \in \Gamma_{\mathcal{A}} .
$$

Each polytope $\mathcal{A}$ comes with a representation $\rho_{\mathcal{A}}: \pi_{1}\left(M, x_{0}\right) \times \operatorname{Nov}(\mathcal{A}) \rightarrow \operatorname{Nov}(\mathcal{A}), \quad \rho_{\mathcal{A}}(\eta, \lambda)=t_{0}^{-\Phi_{a_{0}}(\eta)} \cdots t_{k}^{-\Phi_{a_{k}}(\eta)} \cdot \lambda$. Sometimes, we will write out $\Phi_{a_{l}}(\eta)=\int_{\eta} a_{l}$. The importance of the minus sign will become clear in Definition 2.31. To any such representation, one can associate a local coefficient system

$$
\underline{\operatorname{Nov}}(\mathcal{A}): \Pi_{1}(M) \rightarrow \bmod _{\mathrm{Nov}(\mathcal{A})}
$$

\footnotetext{
${ }^{18}$ Note that $A=B$ in $\Gamma_{\mathcal{A}}$ if and only if $\Phi_{a_{l}}(A)=\Phi_{a_{l}}(B)$ for all $l=0, \ldots, k$.
} 
which is unique up to isomorphisms of local coefficients. We briefly recall the construction: ${ }^{19}$ fix a basepoint $x_{0} \in M$ and pick for every $x \in M$ a homotopy class of paths $\left\{\eta_{x}\right\}$ relative to the endpoints $x_{0}$ and $x$. Set

$$
\underline{\operatorname{Nov}}(\mathcal{A})(x):=\operatorname{Nov}(\mathcal{A}), \quad \forall x \in M .
$$

For every homotopy class $\{\gamma\}$ relative to the endpoints $x$ and $y$, we first define a loop

$$
\underline{\gamma}:=\eta_{x} * \gamma * \eta_{y}^{-1}: S^{1} \rightarrow M,
$$

based at $x_{0}$, and then define ${ }^{20}$

$$
\underline{\operatorname{Nov}}(\mathcal{A})(\gamma): \underline{\operatorname{Nov}}(\mathcal{A})(y) \longrightarrow \underline{\operatorname{Nov}}(\mathcal{A})(x), \underline{\operatorname{Nov}}(\mathcal{A})(\gamma)(\cdot):=\rho_{\mathcal{A}}(\underline{\gamma}, \cdot) .^{20}
$$

Taking a closer look at (22) reveals that the Novikov ring isomorphism $\underline{\operatorname{Nov}}(\mathcal{A})(\gamma)$ is given by multiplication with

$$
t_{0}^{-\int_{\underline{\gamma}} a_{0}} \cdots t_{k}^{-\int_{\underline{\gamma}} a_{k}} \in \mathbb{Z}\left[\Gamma_{\mathcal{A}}\right] \subseteq \operatorname{Nov}(\mathcal{A})
$$

hence, we may also view it as a $\mathbb{Z}\left[\Gamma_{\mathcal{A}}\right]$-module isomorphism.

Remark 2.30. Usually, local coefficients are considered to take values in the category of abelian groups and are often called "bundle of abelian groups". Mapping into $\bmod _{\operatorname{Nov}(\mathcal{A})}$ will allow us to obtain actual Novikov-module isomorphisms at times where working with bundle of abelian groups would merely grant group isomorphisms.

With this, we define the anticipated twisted Novikov complexes.

Definition 2.31. Let $\theta: \mathcal{A} \rightarrow \Omega^{1}(M)$ be a section as above. We define the twisted Novikov chain complex groups by

$$
\mathrm{CN}_{\bullet}\left(\theta_{a}, \underline{\operatorname{Nov}}(\mathcal{A})\right):=\bigoplus_{x \in Z\left(\theta_{a}\right)} \operatorname{Nov}(\mathcal{A})\langle x\rangle, \quad \forall a \in \mathcal{A} .
$$

The twisted boundary operator $\underline{\partial}=\underline{\partial}_{\theta_{a}}$ is defined by

$$
\begin{aligned}
\underline{\partial}(\lambda x) & :=\sum_{y, \gamma \in \underline{\mathcal{M}}\left(x, y ; \theta_{a}\right)} \underline{\operatorname{Nov}(\mathcal{A})\left(\gamma^{-1}\right)(\lambda) y} \\
& =\sum_{y, \gamma \in \underline{\mathcal{M}}\left(x, y ; \theta_{a}\right)} t_{0}^{\int_{\underline{\gamma}} a_{0}} \cdots t_{k}^{\int_{\underline{\gamma}} a_{k}} \cdot \lambda y, \quad \forall \lambda \in \operatorname{Nov}(\mathcal{A}) .
\end{aligned}
$$

The twisted chain complexes

$$
\left(\mathrm{CN}_{\bullet}\left(\theta_{a}, \underline{\operatorname{Nov}}\left(\left.\mathcal{A}\right|_{\mathcal{B}}\right)\right), \underline{\partial}_{\theta_{a}}\right), \quad \forall a \in \mathcal{A}
$$

are defined analogously. The corresponding twisted Novikov homologies are denoted by

$$
\mathrm{HN}_{\bullet}\left(\theta_{a}, \underline{\operatorname{Nov}}(\mathcal{A})\right) \text { and } \mathrm{HN}_{\bullet}\left(\theta_{a}, \underline{\operatorname{Nov}}\left(\left.\mathcal{A}\right|_{\mathcal{B}}\right)\right), \quad \forall a \in \mathcal{A}, \mathcal{B} \subseteq \mathcal{A} \text {. }
$$

\footnotetext{
${ }^{19}$ This stems from the construction of a category equivalence between the fundamental groupoid and the fundamental group of a sufficiently nice topological space. This procedure allows to switch back and forth between local coefficients and representations, see [10, Page 17], [1, Chapter 2] and [3] for more details.

${ }^{20}$ Note that $\Gamma(\gamma * \eta)=\Gamma(\gamma) \circ \Gamma(\eta)$.
} 
For every flow line $\gamma \in \underline{\mathcal{M}}\left(x, y ; \theta_{a}\right)$, we call

$$
\underline{\operatorname{Nov}}(\mathcal{A})\left(\gamma^{-1}\right)=t_{0}^{\int_{\underline{\underline{\gamma}}} a_{0}} \cdots t_{k}^{\int_{\underline{\underline{\gamma}}} a_{k}}
$$

the Novikov twist of $\gamma$.

Remark 2.32. A priori it is not clear that $\underline{\partial}$ maps into the prescribed chain complex. We will prove this in the next subsection; see Proposition 2.34.

The Novikov twist of $\gamma$ determines the lifting behavior of $\gamma$. Indeed, if $\gamma_{0}, \gamma_{1}$ are two paths from $x$ to $y$ with unique lifts $\tilde{\gamma}_{1}(0)=\tilde{\gamma}_{0}(0)=\tilde{x}$, then

$$
\begin{aligned}
& \tilde{\gamma}_{0}(1)=\tilde{\gamma}_{1}(1) \Longleftrightarrow \int_{\gamma_{0} * \gamma_{1}^{-1}} a_{l}=0, \forall l=0, \ldots, k \\
& \Longleftrightarrow \int_{\gamma_{0}} a_{l}=\int_{\gamma_{1}} a_{l}, \forall l=0, \ldots, k \\
& \Longleftrightarrow \int_{\underline{\gamma_{0}}} a_{l}=\int_{\underline{\gamma_{1}}} a_{l}, \forall l=0, \ldots, k \\
& \Longleftrightarrow t_{0}^{\int_{\underline{\gamma_{0}}} a_{0}} \cdots t_{k}^{\int_{\underline{\gamma_{0}}} a_{k}}=t_{0}^{\int_{{\underline{\gamma_{1}}}_{1}} a_{0}} \cdots t_{k}^{\int_{{\underline{\gamma_{1}}}_{1}} a_{k}} .
\end{aligned}
$$

This will be key in the next subsection.

As the next examples show, we recover the twisted Morse chain complex and its homology as a special case.

Example 2.33. Pick $\mathcal{A}=\langle 0, a\rangle$ and a section $\theta: \mathcal{A} \rightarrow \Omega^{1}(M)$. Then

$$
\operatorname{Nov}\left(\left.\mathcal{A}\right|_{a}\right) \cong \operatorname{Nov}(a) \cong \Lambda_{a}
$$

thanks to Proposition 2.27 and Example 2.3. Furthermore, the twisted chain complex

$$
\mathrm{CN} \bullet\left(\theta_{0}, \underline{\operatorname{Nov}}\left(\left.\mathcal{A}\right|_{a}\right)\right)=\mathrm{CN} \bullet\left(\theta_{0}, \underline{\operatorname{Nov}}(a)\right)
$$

agrees with the twisted Morse complex

$$
\text { CM• }(h, \underline{\operatorname{Nov}}(a)) \text {, where } \theta_{0}=d h .
$$

\subsection{Comparing twisted and polytope complexes}

In general, the same issues as in Sect. 2.2 arises when trying to prove that the twisted Novikov complexes are well defined: it is not clear whether $\underline{\partial}$ maps into the desired chain complex. This is a non-issue in the special case of $\theta_{0}=d h$, i.e., twisted Morse homology - the reason is that the 0-dimensional moduli spaces $\underline{\mathcal{M}}(x, y ; h)$ are compact, hence finite. Compare this to [20]. In the following however, we will see that the twisted chain groups can always be identified with $\mathrm{CN}_{\bullet}\left(\theta_{a}, \mathcal{A}\right)$, so that $\underline{\partial}$ and $\partial$ agree, which then resolves the well-definedness issue by Theorem 2.14. In other words, the twisted chain complex is an equivalent description of the polytope chain complex.

Proposition 2.34. Let $\theta: \mathcal{A} \rightarrow \Omega^{1}(M)$ be a section as above. Then, the twisted and polytope Novikov chain groups are isomorphic

$$
\mathrm{CN}_{\bullet}\left(\theta_{a}, \underline{\operatorname{Nov}}(\mathcal{A})\right) \longleftrightarrow \mathrm{CN}_{\bullet}\left(\theta_{a}, \mathcal{A}\right),
$$


as Novikov modules. The isomorphism is preserved upon restrictions $\left.\mathcal{A}\right|_{\mathcal{B}}$, and $\partial=\underline{\partial}$ up to the identification (23).

In particular, the twisted Novikov chain complexes are well defined with $\mathrm{HN}_{\bullet}\left(\theta_{a}, \underline{\operatorname{Nov}}(\mathcal{A})\right)=\mathrm{HN}_{\bullet}\left(\theta_{a}, \mathcal{A}\right)$ and $\mathrm{HN}_{\bullet}\left(\theta_{a}, \underline{\operatorname{Nov}}\left(\left.\mathcal{A}\right|_{\mathcal{B}}\right)\right)=\mathrm{HN}_{\bullet}\left(\theta_{a},\left.\mathcal{A}\right|_{\mathcal{B}}\right)$, for all $a \in \mathcal{A}$ and subpolytopes $\mathcal{B} \subseteq \mathcal{A}$.

Proof. First of all recall that we can view the $i$-th polytope Novikov chain groups as finitely generated Novikov modules by fixing a finite set of preferred lifts $\tilde{x}_{m} \in \pi^{-1}\left(x_{m}\right)$, for each zero $x_{m}$ of $\theta_{a}$ of index $i$

$$
\mathrm{CN}_{i}\left(\theta_{a}, \mathcal{A}\right) \cong \bigoplus_{m} \Lambda_{\mathcal{A}}\left\langle\tilde{x}_{m}\right\rangle, \quad \text { as Novikov ring modules. }
$$

Since $\Lambda_{\mathcal{A}} \cong \operatorname{Nov}(\mathcal{A})$ (cf. Proposition 2.29), we end up with

$$
\mathrm{CN}_{i}\left(\theta_{a}, \mathcal{A}\right) \cong \bigoplus_{m} \Lambda_{\mathcal{A}}\left\langle\tilde{x}_{m}\right\rangle \cong \bigoplus_{m} \operatorname{Nov}(\mathcal{A})\left\langle x_{m}\right\rangle=\mathrm{CN}_{i}\left(\theta_{a}, \underline{\operatorname{Nov}}(\mathcal{A})\right) .
$$

Both boundary operators $\partial$ and $\underline{\partial}$ are $\Lambda_{\mathcal{A}^{-}}$and $\operatorname{Nov}(\mathcal{A})$-linear, and thus, it suffices to compare $\partial \tilde{x}_{m}$ and $\underline{\partial} x_{m}$. On the one hand, we have

$$
\partial \tilde{x}_{m}=\sum_{n} \lambda_{m, n} \tilde{y}_{n}, \text { with } \lambda_{m, n}=\sum_{A \in \Gamma_{\mathcal{A}}} \# \text { alg } \underline{\mathcal{M}}\left(\tilde{x}_{m}, A \tilde{y}_{n} ; \tilde{f}_{\theta_{a}}\right) A \in \Lambda_{\mathcal{A}} \text {, }
$$

and on the other hand

$$
\underline{\partial} x_{m}=\sum_{n}\left(\sum_{\gamma \in \underline{\mathcal{M}}\left(x_{m}, y_{n}, \theta_{a}\right)} t_{0}^{\int_{\underline{\gamma}} a_{0}} \cdots t_{k}^{\int_{\underline{\gamma}} a_{k}}\right) y_{n} .
$$

In the previous subsection, we have seen that the Novikov twist $t_{0}^{\int_{\underline{\gamma}} a_{0}} \cdots t_{k}^{\int_{\underline{\gamma}} a_{k}}$ of $\gamma$ uniquely determines the lifting behavior of $\gamma$. Thus, if $\tilde{\gamma}$ denotes the unique lift which starts at $\tilde{x}_{n}$ and ends at some $\tilde{y}$, we get

$$
\tilde{y}=A \tilde{y}_{n},
$$

with $A \in \Gamma_{\mathcal{A}} \subset \Lambda_{\mathcal{A}}$ corresponding to $t_{0}^{\int_{\underline{\gamma}} a_{0}} \cdots t_{k}^{\int_{\underline{\gamma}}} a_{k} \in \operatorname{Nov}(\mathcal{A})$. This proves that (24) and (25) agree up to identifying the respective isomorphic Novikov rings. The same proof also shows that the restricted complexes associated with $\left.\mathcal{A}\right|_{\mathcal{B}}$ agree.

With the identification of twisted and polytope complexes at hand, we can invoke Theorem 2.14 (recall that we already assumed that $\theta$ is perturbed accordingly) and deduce that the twisted chain complex is well defined. By the first part, it follows that the corresponding homologies agree. This finishes the proof.

Remark 2.35. The twisted complex can be used to deduce properties of the polytope complex and vice versa. For instance, trying to prove that $\partial^{2}=0$ is equivalent to proving $\underline{\partial}^{2}=0$, which has a far more pleasant proof - the reason is that the Novikov twists are nicely behaved with respect to the compactification of the moduli spaces, see for instance [20, Proposition 1]. 


\section{Applications of the main theorem}

\subsection{The 0-vertex trick and the Morse-Eilenberg Theorem}

All the applications we are about to present boil down to what we call the 0 vertex trick. The idea is to relate the polytope chain groups to twisted Morse chain groups by extending the underlying polytope $\mathcal{A}$ with $0 \in H_{\mathrm{dR}}^{1}(M)$ as an additional vertex, and then using the Main Theorem 2.24. This trick suffices to prove the (twisted) Novikov Morse Homology Theorem (cf. Theorem 3.3 and Corollary 3.4).

For the polytope Novikov Principle (cf. Theorem 3.6), we shall need a Morse variant of the Eilenberg Theorem [3, Theorem 24.1], which has been proven in [1, Theorem 2.21]. We will state and prove a slightly stronger version in the context of Novikov theory down below; see Lemma 3.2.

Lemma 3.1. (0-vertex trick) Let $\theta: \mathcal{A} \rightarrow \Omega^{1}(M)$ be a section, $\mathcal{B} \subseteq \mathcal{A}$ a subpolytope, and $\mathcal{A}^{0}$ the polytope spanned by the vertices of $\mathcal{A}$ and $0{ }^{21}$ Let $\theta^{0}: \mathcal{A}^{0} \rightarrow \Omega^{1}(M)$ be a section extending $\theta$. Then

$$
\mathbb{Z}\left[\Gamma_{\mathcal{A}}\right]=\mathbb{Z}\left[\Gamma_{\mathcal{A}^{0}}\right] \quad \text { and } \quad \underline{\operatorname{Nov}}\left(\left.\mathcal{A}\right|_{\mathcal{B}}\right)=\underline{\operatorname{Nov}}\left(\left.\mathcal{A}^{0}\right|_{\mathcal{B}}\right) .
$$

In particular, there exists a perturbed section $\vartheta^{0}: \mathcal{A}^{0} \rightarrow \Omega^{1}(M)$, such that $\mathrm{CN} \bullet\left(\vartheta_{a}^{0},\left.\mathcal{A}\right|_{\mathcal{B}}\right)=\mathrm{CN}_{\bullet}\left(\vartheta_{a}^{0},\left.\mathcal{A}^{0}\right|_{\mathcal{B}}\right) \simeq \mathrm{CN}_{\bullet}\left(\vartheta_{0}^{0},\left.\mathcal{A}^{0}\right|_{\mathcal{B}}\right)=\mathrm{CM}_{\bullet}\left(h, \underline{\operatorname{Nov}}\left(\left.\mathcal{A}\right|_{\mathcal{B}}\right)\right), \quad \forall a \in \mathcal{A}$, as chain complexes, where $d h=\vartheta_{0}^{0}$.

Proof. Since $\operatorname{ker}(0)=\pi_{1}(M)$, adding 0 as vertex does not affect the underlying abelian cover, i.e., $\widetilde{M}_{\mathcal{A}}=\widetilde{M}_{\mathcal{A}^{0}}$, also see Example 2.3. Thus, the deck transformation groups $\Gamma_{\mathcal{A}}$ and $\Gamma_{\mathcal{A}^{0}}$ are equal and so are the respective group rings. The finiteness conditions for $\operatorname{both} \operatorname{Nov}\left(\left.\mathcal{A}\right|_{\mathcal{B}}\right)$ and $\operatorname{Nov}\left(\left.\mathcal{A}^{0}\right|_{\mathcal{B}}\right)$ are determined by the subpolytope $\mathcal{B} \subseteq \mathcal{A}$, and thus, by the group ring equality, we also deduce

$$
\operatorname{Nov}\left(\left.\mathcal{A}\right|_{\mathcal{B}}\right)=\operatorname{Nov}\left(\left.\mathcal{A}^{0}\right|_{\mathcal{B}}\right)
$$

The equality as local coefficient systems then also follows by observing that the period homomorphism $\Phi_{0}$ is identically zero, and hence, $t^{\Phi_{0}(A)}=1$ for all $A \in \Gamma_{\mathcal{A}^{0}}$. Pick $\vartheta^{0}$ as in Theorem 2.14 (or Corollary 2.17). The first chain polytope equality follows from the Novikov rings being equal and the chain homotopy equivalence stems from the Main Theorem 2.24. The last equality follows from Example 2.33 and the equality of local coefficient systems above.

We conclude the subsection by stating and proving a chain-level Morse variant of the Eilenberg Theorem [1, Theorem 2.21]

Lemma 3.2. (Morse-Eilenberg Theorem) Let $h: M \rightarrow \mathbb{R}$ be Morse function, $\mathcal{A}$ a polytope, and $\mathcal{B} \subseteq \mathcal{A}$ a subpolytope. Then

$$
\mathrm{CM}_{\bullet}(\tilde{h}) \otimes_{\mathbb{Z}\left[\Gamma_{\mathcal{A}}\right]} \operatorname{Nov}\left(\left.\mathcal{A}\right|_{\mathcal{B}}\right) \cong \mathrm{CM}_{\bullet}\left(h, \underline{\operatorname{Nov}}\left(\left.\mathcal{A}\right|_{\mathcal{B}}\right)\right)
$$

as chain complexes over $\operatorname{Nov}\left(\left.\mathcal{A}\right|_{\mathcal{B}}\right)$, where $\tilde{h}=h \circ \pi$.

\footnotetext{
${ }^{21}$ Note that if 0 is already contained in $\mathcal{A}$, then $\mathcal{A}^{0}=\mathcal{A}$.
} 
The proof of [1, Theorem 2.21] constructs a group chain isomorphism $\Psi$ and a careful inspection reveals that $\Psi$ defines a Novikov-module chain isomorphism when working with the according local coefficient system $\underline{\operatorname{Nov}}\left(\left.\mathcal{A}\right|_{\mathcal{B}}\right)$. Nevertheless, we decided to give a full proof of Lemma 3.2 using the tools developed in the previous sections.

Proof of Lemma 3.2. First of all, observe that $\mathrm{CM}_{\bullet}(\tilde{h})$ is a finite $\mathbb{Z}\left[\Gamma_{\mathcal{A}}\right]$ module

$$
\mathrm{CM}_{\bullet}(\tilde{h})=\bigoplus_{m} \mathbb{Z}\left[\Gamma_{\mathcal{A}}\right]\left\langle\tilde{x}_{m}\right\rangle
$$

Here, $\left\{\tilde{x}_{m}\right\}$ denotes a finite set of preferred lifts as in the proof of Proposition 2.34. Define

$\Psi: \mathrm{CM}_{\bullet}(\tilde{h}) \otimes_{\mathbb{Z}\left[\Gamma_{\mathcal{A}}\right]} \operatorname{Nov}\left(\left.\mathcal{A}\right|_{\mathcal{B}}\right) \longrightarrow \mathrm{CM}_{\bullet}\left(h, \underline{\operatorname{Nov}}\left(\left.\mathcal{A}\right|_{\mathcal{B}}\right)\right), \Psi\left(\tilde{x}_{m} \otimes \lambda\right)=\lambda\left\langle x_{m}\right\rangle$.

By the above observation, $\Psi$ is a well defined $\mathbb{Z}\left[\Gamma_{\mathcal{A}}\right]$-linear map. It is clear that $\Psi$ is surjective. For injectivity, we observe that

$$
\Psi\left(\tilde{x}_{m} \otimes \lambda\right)=\Psi\left(\tilde{x}_{n} \otimes \mu\right) \Longleftrightarrow \lambda\left\langle x_{m}\right\rangle=\mu\left\langle x_{n}\right\rangle .
$$

Therefore, we must have $\lambda=\mu$ and $x_{m}=x_{n}$, and hence, $\tilde{x}_{m}=\tilde{x}_{n}$-recall that we are working with a preferred set of critical points in each fiber. This proves injectivity.

Next, we show that $\Psi$ is $\operatorname{Nov}\left(\left.\mathcal{A}\right|_{\mathcal{B}}\right)$-linear. ${ }^{22} \operatorname{Pick} \lambda, \mu \in \operatorname{Nov}\left(\left.\mathcal{A}\right|_{\mathcal{B}}\right)$ and observe

$\Psi\left(\mu \cdot\left(\tilde{x}_{m} \otimes \lambda\right)\right)=\Psi\left(\tilde{x}_{m} \otimes(\mu \cdot \lambda)\right)=(\mu \cdot \lambda)\left\langle x_{m}\right\rangle=\mu \cdot\left(\lambda\left\langle x_{m}\right\rangle\right)=\mu \cdot \Psi\left(\tilde{x}_{m} \otimes \lambda\right)$.

Hence, $\Psi$ is a Novikov-linear isomorphism. We are only left to show $\underline{\partial} \circ \Psi=\Psi \circ$ $\left(\partial^{M} \otimes \mathrm{id}\right)$. Recall that $\partial^{M}$ is defined by counting Novikov-Morse trajectories of $\tilde{h}$ on $\widetilde{M}_{\mathcal{A}}$. In particular, the boundary operator $\partial$ on $\mathrm{CN}_{\bullet}\left(d h,\left.\mathcal{A}\right|_{\mathcal{B}}\right)$ agrees with $\partial^{M}$ on $\operatorname{Crit}(\tilde{h})$. Let us adopt the notation of the proof of Proposition 2.34 and write

$$
\partial^{M} \tilde{x}_{m}=\partial \tilde{x}_{m}=\sum_{n} \lambda_{m, n} \tilde{y}_{n}, \quad \lambda_{m, n} \in \mathbb{Z}\left[\Gamma_{\mathcal{A}}\right] \cdot{ }^{23}
$$

23 Therefore

$$
\Psi \circ\left(\partial^{M} \otimes \mathrm{id}\right) \tilde{x}_{m} \otimes \lambda=\Psi\left(\sum_{n} \lambda_{m, n} \tilde{y}_{n} \otimes \lambda\right)=\sum_{n} \lambda_{m, n} \cdot \lambda\left\langle y_{n}\right\rangle,
$$

by Novikov linearity of $\Psi$. On the other hand, Proposition 2.34 says that up to identifying $\tilde{x}_{m}$ and $x_{m}$, we have $\partial \tilde{x}_{m}=\underline{\partial} x_{m}$, and thus

$$
\underline{\partial} \circ \Psi\left(\tilde{x}_{m} \otimes \lambda\right)=\underline{\partial}\left(\lambda\left\langle x_{m}\right\rangle\right)=\lambda \underline{\partial} x_{m}=\lambda \cdot \sum_{n} \lambda_{m, n}\left\langle y_{n}\right\rangle .
$$

However, $\operatorname{Nov}\left(\left.\mathcal{A}\right|_{\mathcal{B}}\right)$ is a commutative ring, and hence, we conclude the chain property of $\Psi$ and thus that $\Psi$ defines a Novikov-linear chain isomorphism.

\footnotetext{
${ }^{22}$ We are implicitly identifying $\Lambda_{\left.\mathcal{A}\right|_{\mathcal{B}}}=\operatorname{Nov}\left(\left.\mathcal{A}\right|_{\mathcal{B}}\right)$, see Proposition 2.27.

${ }^{23}$ Sanity check: $\lambda_{m, n}=\sum_{\mathcal{A} \in \Gamma_{\mathcal{A}}} \#$ alg $\underline{\mathcal{M}}\left(\tilde{x}_{m}, A \tilde{y}_{n} ; \tilde{h}\right) A$, but $\bigcup_{A \in \Gamma_{\mathcal{A}}} \underline{\mathcal{M}}\left(\tilde{x}_{m}, A \tilde{y}_{n} ; \tilde{h}\right) \cong$ $\underline{\mathcal{M}}\left(x_{m}, y_{n} ; h\right)$ and the latter is finite.
} 


\subsection{The twisted Novikov Morse Homology Theorem}

Using the results developed in Sect. 2 and the 0-vertex trick (cf. Lemma 3.1), we are going to prove:

Theorem 3.3. Let $f$ be a Morse function and $a \in H_{\mathrm{dR}}^{1}(M)$ a cohomology class. Then, for every Morse representative $\alpha \in a$, there exists a chain homotopy equivalence

$$
\mathrm{CN}_{\bullet}(\alpha) \simeq \mathrm{CM}_{\bullet}(f, \underline{\mathrm{Nov}}(a))
$$

of Novikov modules.

One can prove that the twisted Morse homology computes singular homology with coefficients $\underline{\operatorname{Nov}}(a)$; see [1, Theorem 4.1]. ${ }^{24}$ Combining this with Theorem 3.3 and taking the homology then shows:

Corollary 3.4. (Twisted Novikov Homology Theorem) For any cohomology class $a \in H_{\mathrm{dR}}^{1}(M)$, there exists an isomorphism

$$
\mathrm{HN}_{\bullet}(a) \cong \mathrm{H}_{\bullet}(M, \underline{\mathrm{Nov}}(a))
$$

of Novikov modules.

Remark 3.5. This is a slightly different incarnation of the classical Novikov Morse Homology Theorem as Corollary 3.4 relates the Novikov homology to twisted singular homology rather than equivariant singular homology. Moreover, as the proof will show, we do not invoke the Eilenberg Theorem (or its Morse analogue from the previous subsection) and instead produce a direct connection between the Novikov complex and the twisted Morse complex via the 0 -vertex trick - this chain of arguments appears to be novel.

Proof of Theorem 3.3. Pick $0, a$ in $H_{\mathrm{dR}}^{1}(M)$, set $\mathcal{A}:=\langle 0, a\rangle$, and consider any section

$$
\theta: \mathcal{A} \rightarrow \Omega^{1}(M)
$$

Up to perturbing $\theta$, we may assume that $\theta$ is a section $\vartheta^{0}$ (note that here $\mathcal{A}^{0}=\mathcal{A}$ ) as in Lemma 3.1. In particular, setting $\mathcal{B}=\langle a\rangle$ and invoking Lemma 3.1 , we get a Novikov chain homotopy equivalence

$$
\mathrm{CN}_{\bullet}\left(\theta_{a},\left.\mathcal{A}\right|_{a}\right) \simeq \mathrm{CM}_{\bullet}\left(h, \underline{\operatorname{Nov}}\left(\left.\mathcal{A}\right|_{a}\right)\right) .
$$

From Example 2.33, we deduce that $\underline{\operatorname{Nov}}\left(\left.\mathcal{A}\right|_{a}\right)=\underline{\operatorname{Nov}}(a)$, and therefore, we obtain

$$
\mathrm{CN}_{\bullet}\left(\theta_{a}\right) \simeq \mathrm{CM}_{\bullet}(h, \underline{\operatorname{Nov}}(a))
$$

as Novikov modules. Twisted Morse homology, just as ordinary Morse homology, does not depend on the choice of Morse function. Indeed, using continuation methods, one can prove

$$
\mathrm{CN}_{\bullet}(h, \underline{\operatorname{Nov}}(a)) \simeq \mathrm{CM}_{\bullet}(f, \underline{\operatorname{Nov}}(a))
$$

\footnotetext{
${ }^{24}$ The authors mention in the proof of [1, Lemma 6.30] that the isomorphism in [1, Theorem 4.1] is an isomorphism in the category of the underlying local coefficient system, thus a Novikov-module isomorphism in our case.
} 
as Novikov modules. ${ }^{25}$ This proves

$$
\mathrm{CN}_{\bullet}\left(\theta_{a}\right) \simeq \mathrm{CM}_{\bullet}(f, \underline{\operatorname{Nov}}(a))
$$

as Novikov modules. Observing $\theta_{a} \in a$ and taking the homology on both sides complete the proof.

\subsection{A polytope Novikov principle}

Combining the two lemmata from Sect. 3.1 and the Main Theorem 2.24, we will prove a polytope Novikov Principle (see Theorem 3.6). As a corollary, we recover the ordinary Novikov Principle (cf. Corollary 3.8). In fact, the results here also cover those in Sect. 3.2. We opted to keep them apart to emphasize the novelty of the "twisted" approach to the Novikov Homology Theorem (see Remark 3.5).

The polytope Novikov Principle yields a new proof (in the abelian case) to a recent result due to Pajitnov [17, Theorem 5.1].

Theorem 3.6. (Polytope Novikov Principle) Let $\theta: \mathcal{A} \rightarrow \Omega^{1}(M)$ be any section and $\mathcal{B} \subseteq \mathcal{A}$ a subpolytope. Then, there exists a perturbed section $\vartheta: \mathcal{A} \rightarrow$ $\Omega^{1}(M)$, such that

$$
\mathrm{CN}_{\bullet}\left(\vartheta_{a},\left.\mathcal{A}\right|_{\mathcal{B}}\right) \simeq C_{\bullet}\left(\widetilde{M}_{\mathcal{A}}\right) \otimes_{\mathbb{Z}\left[\Gamma_{\mathcal{A}}\right]} \operatorname{Nov}\left(\left.\mathcal{A}\right|_{\mathcal{B}}\right), \quad \forall a \in \mathcal{A}
$$

as Novikov modules.

Here, $C$ • denotes the singular chain complex with $\mathbb{Z}$-coefficients.

Proof of Theorem 3.6. Let $\theta^{0}: \mathcal{A}^{0} \rightarrow \Omega^{1}(M)$ be a section that extends $\theta$ with $\mathcal{A}^{0}$ the polytope generated by the vertices of $\mathcal{A}$ and 0 . By the 0 -vertex trick, i.e., Lemma 3.1, there exists a perturbed section $\vartheta^{0}: \mathcal{A}^{0} \rightarrow \Omega^{1}(M)$, such that

$$
\mathrm{CN}_{\bullet}\left(\vartheta_{a}^{0},\left.\mathcal{A}\right|_{\mathcal{B}}\right) \simeq \mathrm{CM}_{\bullet}\left(h, \underline{\operatorname{Nov}}\left(\left.\mathcal{A}\right|_{\mathcal{B}}\right)\right), \quad \forall a \in \mathcal{A}
$$

as Novikov modules. Combining this with Lemma 3.2, we obtain

$$
\mathrm{CN}_{\bullet}\left(\vartheta_{a}^{0},\left.\mathcal{A}\right|_{\mathcal{B}}\right) \simeq \mathrm{CM}_{\bullet}(\tilde{h}) \otimes_{\mathbb{Z}\left[\Gamma_{\mathcal{A}}\right]} \operatorname{Nov}\left(\left.\mathcal{A}\right|_{\mathcal{B}}\right), \quad \forall a \in \mathcal{A}
$$

as Novikov modules. From standard Morse theory, we know that the Morse chain complex CM. $(\tilde{h})$ is chain homotopy equivalent over $\mathbb{Z}\left[\Gamma_{\mathcal{A}}\right]$ to the singular chain complex $C \bullet\left(\widetilde{M}_{\mathcal{A}}\right)$; see for instance [16, Page 415] and [15, Appendix]. Denote by

$$
i: \mathrm{CM}_{\bullet}(\tilde{h}) \longrightarrow C_{\bullet}\left(\widetilde{M}_{\mathcal{A}}\right), j: C_{\bullet}\left(\widetilde{M}_{\mathcal{A}}\right) \longrightarrow \mathrm{CM}_{\bullet}(\tilde{h})
$$

such a chain homotopy equivalence. Then, one can easily check that $i \otimes$ $\operatorname{id}_{\operatorname{Nov}\left(\left.\mathcal{A}\right|_{\mathcal{B}}\right)}$ and $j \otimes \operatorname{id}_{\operatorname{Nov}\left(\left.\mathcal{A}\right|_{\mathcal{B}}\right)}$ define a Novikov-linear chain homotopy equivalence

$$
\operatorname{CM} \bullet(\tilde{h}) \otimes_{\mathbb{Z}\left[\Gamma_{\mathcal{A}}\right]} \operatorname{Nov}\left(\left.\mathcal{A}\right|_{\mathcal{B}}\right) \simeq C \bullet\left(\widetilde{M}_{\mathcal{A}}\right) \otimes_{\mathbb{Z}\left[\Gamma_{\mathcal{A}}\right]} \operatorname{Nov}\left(\left.\mathcal{A}\right|_{\mathcal{B}}\right) \cdot{ }^{26}
$$

\footnotetext{
${ }^{25}$ See the proof of [1, Theorem 3.9] for more details.
} 
${ }^{26}$ Hence

$\mathrm{CN} \bullet\left(\vartheta_{a}^{0},\left.\mathcal{A}\right|_{\mathcal{B}}\right) \simeq \mathrm{CM} \bullet(\tilde{h}) \otimes_{\mathbb{Z}\left[\Gamma_{\mathcal{A}}\right]} \operatorname{Nov}\left(\left.\mathcal{A}\right|_{\mathcal{B}}\right) \simeq C \bullet\left(\widetilde{M}_{\mathcal{A}}\right) \otimes_{\mathbb{Z}\left[\Gamma_{\mathcal{A}}\right]} \operatorname{Nov}\left(\left.\mathcal{A}\right|_{\mathcal{B}}\right), \quad \forall a \in \mathcal{A}$

Setting $\vartheta:=\left.\vartheta^{0}\right|_{\mathcal{A}}$ finishes the proof.

Remark 3.7. If one is interested in a particular Morse form $\omega$, then the following improvement can be made: let $(\omega, g)$ be Morse-Smale and assume that $\mathcal{A}$ is a polytope around $[\omega]$ that admits a section $\theta: \mathcal{A} \rightarrow \Omega^{1}(M)$ sufficiently close to $(\omega, g)$ in the sense of Corollary 2.17. Denote by $\vartheta^{\omega}: \mathcal{A} \rightarrow \Omega^{1}(M)$ the associated perturbation with reference pair $(\omega, g)$. The section $\vartheta=\left.\vartheta^{0}\right|_{\mathcal{A}}$ in the proof above might come from an exact reference pair, since 0 could a priori be far away from $\theta$. However, Corollary 2.23 asserts

$$
\mathrm{CN}\left(\vartheta_{a}^{\omega},\left.\mathcal{A}\right|_{\mathcal{B}}\right) \simeq \operatorname{CN}\left(\vartheta_{a},\left.\mathcal{A}\right|_{\mathcal{B}}\right), \quad \forall a \in \mathcal{A} \text {. }
$$

Combining this with $(26)$ for $a=[\omega]$ gives

$$
\mathrm{CN}_{\bullet}\left(\omega,\left.\mathcal{A}\right|_{\mathcal{B}}\right) \simeq C_{\bullet}\left(\widetilde{M}_{\mathcal{A}}\right) \otimes_{\mathbb{Z}\left[\Gamma_{\mathcal{A}}\right]} \operatorname{Nov}\left(\left.\mathcal{A}\right|_{\mathcal{B}}\right)
$$

since $\vartheta_{[\omega]}^{\omega}=\omega$.

In the special case of ordinary Novikov theory, i.e., $\mathcal{A}=\langle a\rangle$ and $\mathcal{A}^{0}=$ $\langle 0, a\rangle$, Theorem 3.6 reduces to the ordinary Novikov Principle.

Corollary 3.8. (Ordinary Novikov Principle) Let $(\alpha, g)$ be Morse-Smale. Then

$$
\mathrm{CN}_{\bullet}(\alpha) \simeq C_{\bullet}\left(\widetilde{M}_{a}\right) \otimes_{\mathbb{Z}\left[\Gamma_{a}\right]} \operatorname{Nov}(a) .
$$

Proof. Set $a=[\alpha]$, pick $\mathcal{A}=\langle a\rangle, \mathcal{B}=\mathcal{A}$ and $\theta: \mathcal{A} \rightarrow \Omega^{1}(M)$ the smooth section defined by $\theta_{a}=\alpha$. The section $\theta$ is obviously close to $(\alpha, g)$, and thus, Theorem 3.6 and Remark 3.7 imply

$$
\mathrm{CN}_{\bullet}(\alpha) \simeq C_{\bullet}\left(\widetilde{M}_{a}\right) \otimes_{\mathbb{Z}\left[\Gamma_{a}\right]} \operatorname{Nov}(a)
$$

as Novikov modules.

Even though it is hidden in the proof above, the main idea is still to use perturbations $\vartheta^{0}: \mathcal{A}^{0} \rightarrow \Omega^{1}(M)$ associated with an exact reference pair $(H, g)$. Recall that these sections $\vartheta^{0}$ are constructed by a "shift-and-scale" procedure, so that each $\vartheta_{a}^{0}$ is dominated by the exact term $\frac{1}{\varepsilon} d H$. This strategy to recover the ordinary Novikov Principle has been known among experts for quite awhile; see [14, Page 302] for a historical account, [13, Page 548] and [9, Theorem 3.5.2]. However, our approach is slightly different as it does not make use of gradient like vector fields.

We conclude the present subsection by explaining how to recover $[17$, Theorem 5.1] from the polytope Novikov principle. For the reader's convenience, we briefly recall Pajitnov's setting, keeping the notation as close as

\footnotetext{
${ }^{26}$ One can also deduce from category theory by observing that the functor $F: \bmod _{\mathbb{Z}[\mathcal{A}]} \rightarrow$ $\bmod _{\operatorname{Nov}\left(\left.\mathcal{A}\right|_{\mathcal{B}}\right)}$,$$
F(O):=O \otimes_{\mathbb{Z}\left[\Gamma_{\mathcal{A}}\right]} \operatorname{Nov}\left(\left.\mathcal{A}\right|_{\mathcal{B}}\right), F(i)=i \otimes \mathrm{id}, \quad O, P \in \operatorname{obj}\left(\bmod _{\mathbb{Z}\left[\Gamma_{\mathcal{A}}\right]}\right), i \in \operatorname{hom}(O, P)
$$

is additive. 
possible to [17]. Fix a Morse-Smale pair $(\omega, g)$ on $M$ and let $p: \widehat{M} \rightarrow M$ be a regular cover, such that $p^{*}[\omega]=0$. Denote by $r$ the rank of $\omega^{27}$ and define $G=\operatorname{Deck}(\widehat{M})$. Viewing the period homomorphism $\Phi_{\omega}$ on $H_{1}(M ; \mathbb{Z})$, we get a splitting

$$
H_{1}(M ; \mathbb{Z}) \cong \mathbb{Z}^{r} \oplus \operatorname{ker}[\omega] .
$$

Pajitnov calls a family of homomorphism

$$
\Psi_{1}, \ldots, \Psi_{r}: \mathbb{Z}^{r} \rightarrow \mathbb{Z}
$$

a $\Phi_{\omega}$-regular family if

- the $\Psi_{i}$ span $\operatorname{hom}_{\mathbb{Z}}\left(\mathbb{Z}^{r}, \mathbb{Z}\right)$ and

- the coordinates of $\Phi_{\omega}: \mathbb{Z}^{r} \rightarrow \mathbb{R}$ in the basis $\Psi_{i}$ are strictly positive.

We shall call $\Psi=\left\{\Psi_{i}\right\}$ a $\Phi_{\omega}$-semi-regular family whenever the first bullet point above is satisfied. One should not be fooled by the length of namethe existence of a semi-regular family is obvious and merely an algebraic statement.

To every (semi)-regular family $\Psi=\left\{\Psi_{1}, \ldots, \Psi_{r}\right\}$, we associate the conical Novikov ring

$$
\widehat{\Lambda}_{\Psi}=\bigcap_{i=1}^{r} \widehat{\mathbb{Z}}[G]^{\Psi_{i}} .
$$

The conical Novikov chain complex $\left(\mathcal{N}_{\bullet}(\omega), \partial\right)$ is defined as

$$
\mathcal{N}_{i}(\omega, \Psi)=\mathcal{N}_{i}(\omega)=\bigoplus_{x \in Z_{i}(\omega)} \widehat{\Lambda}_{\Psi}\langle x\rangle,
$$

where the boundary operator $\partial$ is defined as expected: fix preferred lifts $\hat{x}$ of each $x \in Z(\omega)$ and define the $y$-component of $\partial x$ by the (signed) count of $\hat{f}_{\omega}$-Morse flow lines on the cover $\widehat{M}$ from $\hat{x}$ to $g \circ \hat{y}$ for all $g \in G{ }^{28}$ Pajitnov proves that there always exists a $\Phi_{\omega}$-regular family $\Psi$, so that $\left(\mathcal{N}_{\bullet}(\omega), \partial\right)$ is a well-defined $\widehat{\Lambda}_{\Psi}$-module chain complex and shows:

Theorem 3.9. (Pajitnov 2019, [17]) For any Morse-Smale pair $(\omega, g)$, there exists a $\Phi_{\omega}$-regular family $\Psi$, such that $\mathcal{N}_{\bullet}(\omega, \Psi)=\mathcal{N}_{\bullet}(\omega)$ is a well-defined chain complex. Moreover, for any such $\Psi$, it holds

$$
\mathcal{N}_{\bullet}(\omega) \simeq C_{\bullet}(\widehat{M}) \otimes_{\mathbb{Z}[G]} \widehat{\Lambda}_{\Psi}
$$

as $\widehat{\Lambda}_{\Psi}$ modules.

Using Theorem 3.6, we recover Theorem 3.9 in the abelian case.

Corollary 3.10. Let $(\omega, g)$ be a Morse-Smale pair, $p: \widehat{M} \rightarrow M$ be an abelian regular cover with $p^{*}[\omega]=0$.

\footnotetext{
${ }^{27}$ The rank of a cohomology class $a \in H_{\mathrm{dR}}^{1}(M)$ is defined as $\operatorname{rank}_{\mathbb{Z}}\left(\operatorname{im} \Phi_{a}\right.$ ).

${ }^{28}$ The definition in [17] is slightly more general, as they define the count with respect to any transverse $\omega$-gradient.
} 
Then, there exist a $\Phi_{\omega}$-semi-regular family $\Psi$, a section $\theta: \mathcal{A} \rightarrow \Omega^{1}(M)$ around $[\omega]$ with $\widetilde{M}_{\mathcal{A}}=\widehat{M}$, a subpolytope $\mathcal{B} \subseteq \mathcal{A}$, and a perturbation $\vartheta: \mathcal{A} \rightarrow$ $\Omega^{1}(M)$, such that

$$
\mathrm{CN}_{\bullet}\left(\vartheta_{a},\left.\mathcal{A}\right|_{\mathcal{B}}\right) \simeq C_{\bullet}(\widehat{M}) \otimes_{\mathbb{Z}[G]} \widehat{\Lambda}_{\Psi}, \quad \forall a \in \mathcal{A}
$$

as Novikov modules with $\vartheta_{[\omega]}=\omega$. In particular

$$
\mathrm{CN}_{\bullet}\left(\omega,\left.\mathcal{A}\right|_{\mathcal{B}}\right) \simeq C_{\bullet}(\widehat{M}) \otimes_{\mathbb{Z}[G]} \widehat{\Lambda}_{\Psi}
$$

Proof. First of all, we construct the polytope $\mathcal{A}$ and a small section $\theta: \mathcal{A} \rightarrow$ $\Omega^{1}(M)$ by adapting a rational approximation idea due to Pajitnov [15]; see also [21, Section 4.2]. Consider the splitting from before

$$
H_{1}(M ; \mathbb{Z}) \cong \mathbb{Z}^{r} \oplus \operatorname{ker}[\omega],
$$

where $r$ is the rank of $[\omega]$. Pick $r$-many generators $\gamma_{1}, \ldots, \gamma_{r}$ of the first summand above. By de Rham's Theorem, there are $r$-many pairwise distinct integral classes $a_{1}, \ldots, a_{r}$ dual to $\gamma_{1}, \ldots, \gamma_{r}$, such that

$$
\operatorname{ker}\left(a_{l}\right) \supset \operatorname{ker}[\omega], \quad \forall l=1, \ldots, r .
$$

In particular, we can write

$$
\Phi_{\omega}=\sum_{l=1}^{r} u_{l} \cdot \Phi_{a_{l}}
$$

for a unique vector $\underline{u}=\left(u_{1}, \ldots, u_{r}\right) \in \mathbb{R}^{r}$, and hence, $[\omega]=\sum_{l=1}^{r} u_{l} \cdot a_{l}$. Thus, for our fixed Morse representative $\omega \in[\omega]$, there exist $\alpha_{l} \in a_{l}$ with

$$
\omega=\sum_{l=1}^{r} u_{l} \cdot \alpha_{l} \quad \text { and } \quad \operatorname{ker}[\omega]=\bigcap_{l=1}^{r} \operatorname{ker}\left(a_{l}\right) .
$$

For fixed $\varepsilon>0$, we construct $r$-many rational closed one-forms $\beta_{l}$ that are $\varepsilon$-close to $\omega$ (in the operator norm induced by $g$ ). Pick a sufficiently small vector $\underline{v}^{1}=\underline{v}^{1}(\varepsilon) \in \mathbb{R}^{r}$, such that for

$$
\beta_{1}=\omega+\sum_{l=1}^{r} v_{l}^{1} \cdot \alpha_{l}
$$

we have

$$
\left\|\omega-\beta_{1}\right\| \leq \sum_{l=1}^{r}\left|v_{l}^{1}\right| \cdot\left\|\alpha_{l}\right\|<\varepsilon \quad \text { and } \quad b_{1}:=\left[\beta_{1}\right] \in H^{1}(M ; \mathbb{Q}) .
$$

This is possible, since $\mathbb{Q}$ is dense in $\mathbb{R}$. Define

$$
\beta_{2}=\omega+\sum_{l=1}^{r} v_{l}^{2} \cdot \alpha_{l}, \quad \underline{v}^{2} \in \mathbb{R}^{r}
$$

satisfying the same properties with

$$
\underline{v}^{2}-\underline{v}^{1}=(\underbrace{v_{1}^{2}-v_{1}^{1}}_{\neq 0}, 0, \ldots, 0)
$$


In particular

$$
\beta_{2}-\beta_{1}=\left(v_{1}^{2}-v_{1}^{1}\right) \cdot \alpha_{1},
$$

which implies $b_{1} \neq b_{2}$. Proceeding inductively (e.g. $\underline{v}^{3}$ agreeing with $\underline{v}^{2}$ except for the second entry, etc.), we end up with $r$-many rational one-forms $\beta_{1}, \ldots, \beta_{r}$ satisfying

- $\left\|\omega-\beta_{j}\right\| \leq \sum_{l=1}^{r}\left|v_{l}^{j}\right| \cdot\left\|\alpha_{l}\right\|<\varepsilon$ for all $j=1, \ldots, r$.

- $b_{l} \neq b_{j}$ for all $l \neq j$.

- $\operatorname{ker}\left(b_{l}\right) \supset \operatorname{ker}[\omega]$ for all $l=1, \ldots, r$, by $(27)$.

Since all $b_{l}$ are rational, there exists a positive integer $q \in \mathbb{N}$, so that every cohomology class $q \cdot b_{l}$ is integral. From the bullet points above, we thus conclude that

$$
\Psi:=\left\{\Phi_{q \cdot b_{l}}\right\}
$$

defines a $\Phi_{\omega}$-semi-regular family. Next, set $\mathcal{A}^{-}=\left\langle[\omega], b_{1}, \ldots, b_{r}\right\rangle$ and

$$
\varepsilon=\frac{C_{\omega}}{D \cdot 1000},
$$

cf. Corollary 2.17. It is clear that there exists a section $\theta^{-}: \mathcal{A}^{-} \rightarrow \Omega^{1}(M)$ sending $[\omega]$ to $\omega$ and $b_{l}$ to $\beta_{l}$. In particular

$$
\left\|\theta_{a}^{-}-\omega\right\|<\varepsilon, \quad \forall a \in \mathcal{A}^{-} .
$$

Analogously to the construction of the $\beta_{l}$ 's, one can define rational one-forms close to $\omega$ that do not vanish on $\operatorname{ker}[\omega]$. Including some of those cohomology classes allows us to extend $\mathcal{A}^{-}$to $\mathcal{A}$, so that $\widetilde{M}_{\mathcal{A}}=\widehat{M}$ with a section $\theta: \mathcal{A} \rightarrow$ $\Omega^{1}(M)$ that extends $\theta^{-}$and is still $\varepsilon$-close to $\omega$. By choice of $\varepsilon$, we can invoke Corollary 2.17 and define a perturbed section

$$
\vartheta^{\omega}: \mathcal{A} \rightarrow \Omega^{1}(M)
$$

with $(\omega, g)$ as the underlying reference pair. But now, we are in a position to use Theorem 3.6 and Remark 3.7 with $\mathcal{B}:=\left\langle b_{1}, \ldots, b_{r}\right\rangle$ to obtain

$$
\mathrm{CN}_{\bullet}\left(\vartheta_{a}^{\omega},\left.\mathcal{A}\right|_{\mathcal{B}}\right) \simeq C_{\bullet}(\widehat{M}) \otimes_{\mathbb{Z}[G]} \operatorname{Nov}\left(\left.\mathcal{A}\right|_{\mathcal{B}}\right)=C \bullet(\widehat{M}) \otimes_{\mathbb{Z}[G]} \bigcap_{l=1}^{r} \operatorname{Nov}\left(\left.\mathcal{A}\right|_{b_{l}}\right)
$$

The last equality follows from Lemma 2.7. Note that $\operatorname{Nov}\left(\left.\mathcal{A}\right|_{b_{l}}\right)=\widehat{\mathbb{Z}}[G]^{b_{l}}=$ $\widehat{\mathbb{Z}}[G]^{q \cdot b_{l}}$, and therefore, the Novikov ring on the RHS above does coincide with $\widehat{\Lambda}_{\Psi}$. By definition of the perturbation $\vartheta^{\omega}$, we get $\vartheta_{[\omega]}^{\omega}=\omega$, which finally concludes the proof.

\section{Acknowledgements}

I would like to thank Will Merry for all the helpful discussions and for encouraging me to flesh out the polytope picture in the case of Novikov homology. This work has been supported by the Swiss National Science Foundation (grant \# 182564).

Funding Open Access funding provided by ETH Zurich. 
Open Access. This article is licensed under a Creative Commons Attribution 4.0 International License, which permits use, sharing, adaptation, distribution and reproduction in any medium or format, as long as you give appropriate credit to the original author(s) and the source, provide a link to the Creative Commons licence, and indicate if changes were made. The images or other third party material in this article are included in the article's Creative Commons licence, unless indicated otherwise in a credit line to the material. If material is not included in the article's Creative Commons licence and your intended use is not permitted by statutory regulation or exceeds the permitted use, you will need to obtain permission directly from the copyright holder. To view a copy of this licence, visit http:// creativecommons.org/licenses/by/4.0/.

Publisher's Note Springer Nature remains neutral with regard to jurisdictional claims in published maps and institutional affiliations.

\section{References}

[1] Banyaga, A., Hurtubise, D., Spaeth, P.: Twisted Morse complexes. arXiv:1911.07818 [math.AT] (2019)

[2] Benedetti, G.: The contact property for symplectic magnetic fields on $S^{2}$. Ergod. Theory Dyn. Syst. 36(3), 682-713 (2016)

[3] Eilenberg, S.: Homology of spaces with operators. I. Trans. Am. Math. Soc. 61, 378-417 (1947) [errata 62, 548, 1947]

[4] Farber, M.: Topology of closed one-forms. Mathematical Surveys and Monographs, vol. 108. American Mathematical Society, Providence (2004)

[5] Groman, Y., Merry, W.J.: The symplectic cohomology of magnetic cotangent bundles. arXiv:1809.01085 [math.SG] (2018)

[6] Hofer, H., Salamon, D.A.: Floer homology and Novikov rings. In: The Floer Memorial, Progr. Math., vol. 133, pp. 483-524. Birkhäuser, Basel (1995)

[7] Hutchings, M.: Floer homology of families. I. Algebr. Geom. Topol. 8(1), 435$492(2008)$

[8] Latour, F.: Existence de 1-formes fermées non singulières dans une classe de cohomologie de de Rham. Inst. Hautes Études Sci. Publ. Math. 80, 135-194 (1995)

[9] Li, T. et al.: Topology of closed 1-forms on manifolds with boundary. PhD thesis, Durham University (2009)

[10] May, J.P.: A concise course in algebraic topology. Chicago Lectures in Mathematics. University of Chicago Press, Chicago (1999)

[11] Novikov, S.P.: Multivalued functions and functionals. An analogue of the Morse theory. Dokl. Akad. Nauk SSSR 260(1), 31-35 (1981)

[12] Novikov, S.P.: The Hamiltonian formalism and a multivalued analogue of Morse theory. Uspekhi Mat. Nauk. 37(5(227)), 3-49, 248 (1982)

[13] Ono, K.: Floer-Novikov Cohomology and Symplectic Fixed Points, vol. 3, pp. 545-563 (2005). Conference on Symplectic Topology

[14] Pajitnov, A.V.: On the Novikov complex for rational Morse forms. Annales de la Faculté des sciences de Toulouse: Mathématiques 4, 297-338 (1995) 
[15] Pajitnov, A.V.: Incidence coefficients in the Novikov complex for Morse forms: rationality and exponential growth properties. arXiv:dg-ga/9604004 [dgga] (1996)

[16] Pajitnov, A.V.: Circle-valued Morse theory, De Gruyter Studies in Mathematics, vol. 32. Walter de Gruyter \& Co., Berlin (2006)

[17] Pajitnov, A.V.: On the conical Novikov homology. Eur. J. Math. 6(4), 1303$1341(2020)$

[18] Pellegrini, A.: Dynamical symplectic Novikov homology (2020) (in preparation)

[19] Poźniak, M.: Floer homology, Novikov rings and clean intersections. In: Northern California Symplectic Geometry Seminar, Amer. Math. Soc. Transl. Ser. 2, vol. 196, pp. 119-181. Amer. Math. Soc., Providence (1999)

[20] Ritter, A.F.: Novikov-symplectic cohomology and exact Lagrangian embeddings. Geom. Topol. 13(2), 943-978 (2009)

[21] Schütz, D.: Gradient flows of closed 1-forms and their closed orbits. Forum Math. 14(4), 509-537 (2002)

[22] Sikorav, J.-C.: Homologie de Novikov associée à une classe de cohomologie reelle de degré un. PhD thesis, Université Paris-Sud (Orsay) (1987)

[23] Whitehead, G.W.: Elements of Homotopy Theory, Graduate Texts in Mathematics, vol. 61. Springer, New York (1978)

[24] Whitehead, J.H.C.: Convex regions in the geometry of paths. In: James, I. (ed.) Differential Geometry, pp. 223-232. Pergamon (1962)

[25] Zhang, J.: Symplectic structure perturbations and continuity of symplectic invariants. Algebr. Geom. Topol. 19(7), 3261-3314 (2019)

\author{
Alessio Pellegrini \\ Department of Mathematics \\ ETH Zürich \\ Zurich \\ Switzerland \\ e-mail: alessio.pellegrini@math.ethz.ch
}

Accepted: September 2, 2021. 